\title{
Portfolio formation with preselection using deep learning from long-term financial data
}

Article

Accepted Version

Creative Commons: Attribution-Noncommercial-No Derivative Works 4.0

Wang, W., Li, W., Zhang, N. and Liu, K. (2019) Portfolio formation with preselection using deep learning from long-term financial data. Expert Systems with Applications, 143. 113042. ISSN 0957-4174 doi:

https://doi.org/10.1016/j.eswa.2019.113042 Available at https://centaur.reading.ac.uk/86775/

It is advisable to refer to the publisher's version if you intend to cite from the work. See Guidance on citing.

To link to this article DOI: http://dx.doi.org/10.1016/j.eswa.2019.113042

Publisher: Elsevier

All outputs in CentAUR are protected by Intellectual Property Rights law, including copyright law. Copyright and IPR is retained by the creators or other copyright holders. Terms and conditions for use of this material are defined in the End User Agreement.

www.reading.ac.uk/centaur 
Central Archive at the University of Reading

Reading's research outputs online 
Title : Portfolio formation with preselection using deep learning from long-term financial data

\section{Author names and affiliations:}

Wuyu Wang ${ }^{1}$, Weizi Li ${ }^{2}$, Ning Zhang ${ }^{1}$, Kecheng Liu ${ }^{2}$

${ }^{1}$ School of Information, Central University of Finance and Economics, 39 South College Road, Haidian District, Beijing, P.R. China 100081

${ }^{2}$ Informatics Research Centre, Henley Business School, University of Reading, Reading, Berkshire RG6 6UD, UK

Email addresses: cufe_wwy@163.com (Wuyu Wang),weizi.li@henley.ac.uk (Weizi Li), 717212398@qq.com (Ning Zhang), k.liu@ reading.ac.uk (Kecheng Liu) 
3 Abstract: Portfolio theory is an important foundation for portfolio management which is a well-

4 studied subject yet not fully conquered territory. This paper proposes a mixed method consisting of long short-term memory networks and mean-variance model for optimal portfolio formation in conjunction to the asset preselection, in which long-term dependences of financial time-series data can be captured. The experiment uses a large volume of sample data from UK Stock Exchange 100 Index between March 1994 and March 2019. In the first stage, long short-term memory networks are used to forecast the return of assets and select assets with higher potential returns. After comparing the outcomes of the long short-term memory networks against support vector machine, random forest, deep neural networks and autoregressive integrated moving average model, we discover that long short-term memory networks are appropriate for financial time-series forecasting, to beat the other benchmark models by a very clear margin. In the second stage, based on selected month of each triennium. i.e. potential returns and risks.

Key words: asset preselection, long-term financial data, financial forecasting, portfolio optimisation 


\section{term financial data}

24

\section{Introduction}

Portfolio management is a decision-making process in which an amount of fund is allocated to multiple financial assets, and the allocation weight is constantly changed in order to maximize the return and restrain the risk (Markowitz, 1952). Portfolio theory proposed by Markowitz in 1952, is an important foundation for portfolio management which is a well-studied subject yet not fully conquered territory. There are two issues with portfolio formation. The first one is to select assets with higher revenue, and another one is to determine the value composition of assets in the portfolio to achieve the goal of maximal potential returns with minimal risk. Quantitative approach to the portfolio formation has often been adopted in investment decisions. Based on Markowitz's mean-variance (MV) model, numerous researches have discovered many model extensions and supplemented plentiful reasonable insights about the portfolio formation (Tobin, 1958; Sharpe, 1963; Merton, 1969; Grauer and Hakansson, 1993; Liu and Loewenstein, 2002; Tu and Zhou, 2010; Brown and Smith, 2011; Li et al., 2013; Li et al., 2015; Bodnar et al., 2017).

In the portfolio optimisation process, the expected return on an asset is a crucial factor, which means that a preliminary selection of assets is critical for portfolio management (Guerard Jr et al., 2015). But few researches pay attention to the preselection of assets before forming a portfolio. Asset selection has been a meaningful, but difficult issue in financial investment area. This line of research depends on a long-term volatility of financial time-series data in the past as well as a reliable performance forecasting of assets in the future (Huang, 2012). Traditional statistical methods are not effective in dealing with complex, multi-dimensional and noisy time-series data 
44 (Längkvist et al., 2014; Baek and Kim, 2018), while early machine learning methods, such as support vector machine (SVM), principal component analysis (PCA), and artificial neural network (ANN), are not most suited for learning and storing financial time-series data over a long period (LeCun et al., 2015; Bao et al., 2017). This situation leads to the difficulties of financial assets preselection. In fact, during the investment decision-making process, it would be unsustainable to only apply complex portfolio optimisation methods without high-quality asset input (Deng and Min, 2013).

In the financial market, individual investors usually would like to know the changes in the returns of their investment assets today, the possible trends in the returns tomorrow and which measures should be adopted to help them possess the best portfolio (Zhang et al., 2018). Therefore, incorporating forecasting theory into the portfolio formation will be promising in financial investment (Kolm et al., 2014). Forecasting financial time-series is always regarded as one of the most challenging tasks because of the dynamic, nonlinear, unstable and complex nature with longterm fluctuations of the financial market (Chen and Hao, 2018; Paiva et al, 2019). But a reliable investment decision should rely on long-term observations and patterns of behaviour of asset data rather than short-term (Chourmouziadis and Chatzoglou 2016; Chong et al., 2017). In this case, it is necessary to observe the change and volatility of financial data over a long time in the past so as to make a good preparation for future trends forecasting and investment decisions. And numerous widely accepted empirical researches suggest that financial time-series have a memory of a period in the past, thus to some extent, financial markets are predictable. The behaviour of the asset over a long period will significantly influence the risks and returns of a portfolio, and then further affect the investment decisions (Liu and Loewenstein, 2002). However, this important 
point is always ignored by current researches. For instance, some apply early machine learning methods, GA (Huang, 2012), SVM (Huang, 2012; Paiva et al., 2019), to predict and select good

68 assets, but fail to capture long-term dependencies of financial time-series data. To overcome this limitation, we present a novel method for portfolio formation in conjunction to the asset preselection, in which long-term dependences of financial time-series data are duly considered.

The primary purpose of this paper is to construct an investment decision-making model for individual investors that combines the deep learning LSTM method which concentrates on capturing the long-term dependencies of the returns on assets and the Markowitz's MV method to form optimal portfolios. In this respect, our study has two primary contributions which fill the gaps in existing literature. Firstly, this paper develops a novel method consisting of long short-term memory networks and mean-variance model (LSTM+MV) for optimal portfolio formation. This method considers the long-term dependences on the fluctuations of financial market and captures long-time change patterns of company stocks from the time-series data. To show the benefit of the proposed method in terms of the prediction, early machine learning and statistical models are used in our experiments as baselines to compare with the LSTM networks. Secondly, our proposed model explores in-depth the preselection process of assets before optimal portfolio formation, which guarantees high-quality inputs to the optimal portfolio. Unlike the majority of the methods which aim to improve the existing portfolio management models, this paper focuses on the preliminary phase of portfolio construction, i.e. the preselection of assets. Meanwhile our work provides practical guidance for investors in making better investment decisions. Specifically, the systematic approach present in current paper is able to help decide which assets should be part of the portfolio and the value composition of assets in the portfolio. 

of modern portfolio theory and summarise empirical work that has used deep learning to solve issues corresponding to financial time-series data. In Section 3, we describe our methodology in detail, i.e. data source, input variable selection, the proposed model architecture. In Section 4, we present the results of the experiments and explain the results appropriately. In Section 5, we discuss our key findings, implications for theory and practice, also future work.

\section{Theoretical background}

\subsection{Modern Portfolio Theory}

Markowitz (1952) proposes mean-variance (MV) methodology to solve portfolio selection issue, which initiates the foundation of Modern Portfolio Theory (MPT). He quantifies investment return and risk by expected return and variance, respectively. The main idea of MV methodology is to maximize expected return keeping unchanged variance, or minimize variance keeping unchanged expected return. MPT has been widely accepted and studied by researchers. Tobin (1958) indicates that liquidity preference could determine how much wealth is to be invested in monetary assets, and constructs an effective portfolio combined with risk-free assets as well as a special type of risky assets. Sharpe (1963) puts forward the diagonal model assuming that there is no interrelationship among securities so as to simplify the calculation, which significantly facilitates the development of portfolio theory. Some researchers notice that multi-period portfolio selection should be considered to deal with the complex financial markets. For instance, Merton (1969) extends modern portfolio theory by introducing a continuous-time model in order to achieve the goal of maximal expected utility within a constant planning region. Grauer and Hakansson (1993) apply a discrete-time dynamic investment model to compare the MV and the quadratic 
approximations computing the optimal portfolios. Some researches put several realistic constraints into the Markowitz's MV model. For instance, Liu and Loewenstein (2002) incorporate transaction cost into stock trading strategy to help maximize the investors' wealth utility. Brown and Smith (2011) consider risk aversion, transaction cost, portfolio constraints into MV model and find that

114 it would be difficult to solve portfolio optimisation issues when three more assets are involved.

Moreover, some studies use robust optimisation techniques in portfolio management. Tu and Zhou (2010) involve the financial objectives into Bayesian priors to estimate uncertain parameters and they prove that Bayesian method under the objective-based priors performs better than those under alternative priors in portfolio selection. Under a Bayesian estimation framework, Bodnar et al. (2017) analyse the global minimum variance portfolio and consider investors' prior beliefs into the portfolio decisions. On the basis of random matrix theory, Bodnar et al. (2018) evaluate the global minimum variance portfolio with high-dimensional data to minimize the out-of-sample variance. and $\mathrm{Xu}$ (2013) indicate that there are often fuzzy uncertainty and random uncertainty existing in financial market, hence, they incorporate investors' sentiments and experts' insights into the process of portfolio construction. Assuming that expected rate of returns obeys normal distribution, Li et al. (2013) integrate two constraints, value at risk (VaR) and risk-free assets, into a fuzzy portfolio selection model so as to find a more suitable portfolio. Li et al. (2015) put forward another fuzzy portfolio selection model with background risk to obtain the effective frontier of portfolio.

Recently, with the development of big data and artificial intelligence technology, it is possible to use computers and a large number of calculations to achieve optimal portfolio management. Huang 
as vector regression (SVR), but he ignores risk factor. Based on support vector machine (SVM),

Paiva et al. (2019) classify the assets to achieve a certain return and determine the components of the investment portfolio. Almahdi and Yang (2017) set three optimisation objectives, annualised Sharpe ratio, Sterling ratio and Calmar ratio, respectively, then choose the best performance algorithm to select optimal portfolio. Yunusoglu and Selim (2013) develop expert system (ES) to support portfolio managers for investment decisions. The expert system contains three stages, the first stage is elimination of unacceptable stock. The second stage is to evaluate stock through a comprehensive literature survey and interviews with a domain expert. The last stage is to construct portfolio based on a mixed-integer linear programming model. Their results demonstrate that under the different risk preference, ES performance is not particularly big difference, moreover, ES is more suitable for 6 months, 9 months and 12 months of investment period.

It is obvious that various extensions of Markowitz's MV model help enrich the modern portfolio theory and provide researchers with more research perspectives. And these extensions further confirm that MV model plays an extremely significant role in portfolio management. However, most of the related researches ignores the selection of high-quality assets, the stage before the optimal portfolio formation. Instead, they focus more on how to improve the MV model. Actually, high-quality asset input is a reliable guarantee for optimal portfolio formation during the investment process. In this regard, this paper will continue to adopt the classical MV model, moreover, we will study deeply the preliminary selection of assets in order to provide MV model with better asset inputs. At the same time, different transaction costs will be considered for simulation to visualize the performance of different models. 
In recent years, with the development of big data and artificial intelligence (AI) technology, more and more scholars start to use AI as support for their research solutions and prove that AI methods deal with problem of nonlinear, nonstationary characteristics better than traditional statistical models. For example, a number of researches based on SVM (Paiva et al., 2019), PCA (Chen and Hao, 2018; Zbikowski, 2015), GA or random forest (Li and Xu, 2013; Mousavi, 2014), ANN (Patel et al., 2015; Chong et al., 2017) to classify, predict and optimise complex financial assets. Among these technologies, the deep learning is thought to be an appropriate method for the financial time-series forecasting solution, since it is good at processing complex, high-dimensional data as well as extracting abstract characteristics from mass data without depending on any assumptions.

The deep learning method proposed by Hinton and Salakhutdinov (2006), has become a leading application in the financial area, especially in predicting financial market movement and processing text information. Deep learning architectures mainly include deep neural networks (DNNs), deep belief networks (DBNs), recurrent neural networks (RNNs) and convolutional neural networks (CNNs) (LeCun et al., 2015). Amongst them, DNNs are feedforward networks in which data flows from the input layer to the output layer by their single directional forward links without going backwards (Arévalo et al., 2016). Chong et al. (2017) testify that with regard to future returns prediction, DNN is obviously superior to a linear autoregressive model based on data from Korean stock market. Identifying the correlation between different stocks, Lachiheba and Gouider (2018) come up with a DNN model with special structure to predict the trend of stock returns over the next five minutes and the results manifest that the accuracy is improved to $71 \%$ considerably. 
175 DBNs are composed of multiple layers of latent variables, with connections between the layers but 176 not between units within each layer (Hinton, 2009). Shen et al. (2015) construct a DBN using 177 continuous restricted Boltzmann machines to predict exchange rate and their results show that their method performs better than traditional methods. Unlike feedforward neural networks, RNNs can use their internal states (memory) to process sequences of inputs. For instance, Rather et al. (2015) construct a novel hybrid model constituting autoregressive moving average model, exponential smoothing model and RNN to obtain more accurate returns prediction. Similarly, Long et al. (2019) integrate CNN and RNN into their proposed model entitled "multi-filters neural network" aiming to see the trend of the stock price over time, finally, they verify the prediction accuracy of the model through simulation. Long short-term memory (LSTM) networks are one of classes of recurrent neural networks (RNNs), but it has the advantage to retaining information over a long time-span compared with RNNs (LeCun et al., 2015; Fischer and Krauss, 2018). Kraus and Feuerriegel (2017) analyse the text data using the long short-term memory (LSTM) networks, finally they prove that their method increases the accuracy of the stock price prediction. Fischer and Krauss (2018) take advantage of the LSTM networks to forecast stocks directional movement and their results show that LSTM outperforms some classical machine learning models in this prediction task. Besides, Ding et al. (2015) apply CNNs to predict the short-term and long-term influences of events on stock price movements and they prove that the accuracy of the model outperforms other baseline methods.

It is clear that the deep learning method is able to find complex structures in high-dimensional financial data and acquire features through simple and non-linear modules, and then transform 
on above literature review, it is easy to discover that the majority of the existing studies on predicting assets returns based on deep learning pay more attention to improve the prediction accuracy, however, few of them apply their prediction results to actual financial markets, such as portfolio management, assets selection, or trading strategy, to give investors more practical guidance. Actually, the high accuracy of prediction does not represent the optimal investment strategy. The advantages of deep learning methods in predicting can be very helpful for decision making in financial investments (Saurabh Aggarwal and Somya Aggarwal, 2017). Therefore, how to combine the prediction of deep learning to help choose the optimal investment strategy is a meaningful and promising research direction (Zhang et al., 2018).

\section{Methodology}

\subsection{Data}

The biggest challenge of prediction is to recognise a relation in financial time-series data between the past and the future (Paiva et al., 2019). Since the continuity of financial stock data, the longer the sample data is involved, the more likely it is to capture history information memory (Fischer and Krauss, 2018; Long et al., 2019). Hence, a large amount of long-term data is required in the empirical experiment (Chourmouziadis and Chatzoglou, 2016). In this research, we collect daily stock data from the UK Stock Exchange 100 Index (FTSE 100) from March 1994 until March 2019, covering 25 years. Since the majority of related studies have been conducted over a period of 10 years or less (Kara et al, 2011; Patel et al., 2015; Chen and Hao, 2018), 15 years (Paiva et al., 2019; Almahdi and Yang; 2017), or 25 years (Fischer and Krauss, 2018), our samples spanning 25 years can be considered to provide a sufficiently large volume data to generate statistically significant results. Our sample data involves the historical series of adjusted open prices, close prices, the 
219 highest prices, the lowest prices, and the trading volume of assets. Numerous scholars agree on that 220 holding tens of thousands of different stocks as a portfolio is not realistic for individual investors

221 (Tanaka et al., 2000; Ranguelova, 2001; Kocuk and Cornuéjols, 2018; Almahdi and Yang, 2017).

222 For instance, Tanaka et al. (2000) select 9 securities as the sample to form the optimal portfolio.

223 Almahdi and Yang (2017) construct a five-asset portfolio. Hence, this paper randomly chooses 224 twenty-one stocks from FTSE 100 as sample data, which is sufficiently large for the asset 225 preselection before forming portfolio for individual investors. The names of these sample stocks are "BP” (BP), “Barclays” (BAR), “Tesco" (TES), “Vodafone Group” (VG), “Halma” (HAL), “Johnson Matthey" (JM), "HSBC Holdings" (HH), "Sainsbury J" (SJ), "Marks \& Spencer Group" (MSG), "Astrazeneca" (AST), "British American Tobacco" (BAT), "PEARSON" (PEA), "Relx" (RELX), "SSE” (SSE), "Legal \& General" (LG), "Royal Bank" (RB), "Royal Dutch Shell B" (RDSB), "Sage Group" (SG), "Schroders" (SCH), "Seven Trent” (ST) and "Smiths Group" (SG).

231 Their abbreviations are used for convenience, respectively. Table 1 shows the descriptive statistics of close prices for the 21 stocks selected from FTSE 100. As can be seen, stock AST has the highest daily mean prices: 2923.12 , stock LG has the lowest standard deviation: 65.36, stock VG follows, with 65.81 .

Table 1 Descriptive statistics for sample data

\begin{tabular}{lllll}
\hline Stock & Mean & Std. & Maximum & Minimum \\
\hline TES & 255.66 & 104.65 & 492.0 & 67.33 \\
AST & 2923.12 & 1142.28 & 6317.0 & 658.41 \\
BAR & 318.90 & 141.05 & 710.69 & 47.0 \\
BP & 468.58 & 109.99 & 712.0 & 174.5 \\
BAT & 1808.8 & 1468.73 & 5643.0 & 217.59 \\
HAL & 357.44 & 349.40 & 1648.0 & 81.5 \\
HH & 611.21 & 165.72 & 951.6 & 171.09 \\
JM & 1572.69 & 967.46 & 3823.0 & 263.85 \\
LG & 141.78 & 65.36 & 23.0 & 294.4 \\
MSG & 396.84 & 111.54 & 749.0 & 170.75 \\
\hline
\end{tabular}




\begin{tabular}{lllll}
\hline PEA & 887.68 & 316.77 & 2301.79 & 429.5 \\
RELX & 714.0 & 352.18 & 1782.0 & 348.82 \\
RB & 2076.30 & 1877.32 & 6026.35 & 103.0 \\
RDSB & 1753.62 & 451.63 & 761.02 & 2841.0 \\
SG & 978.12 & 283.06 & 424.34 & 1801 \\
SJ & 343.75 & 77.27 & 594.0 & 214.6 \\
SCH & 1416.98 & 875.33 & 3773.0 & 346.01 \\
ST & 1257.59 & 536.65 & 2553.0 & 487.17 \\
SG & 978.12 & 283.06 & 1801.0 & 424.34 \\
SSE & 984.88 & 433.62 & 1696.0 & 272.5 \\
VG & 158.88 & 65.81 & 408.57 & 32.29 \\
\hline
\end{tabular}

236

237

238

239

240

241

242

243

244

245

246

247

\subsection{LSTM networks}

LSTM networks were introduced by Hochreiter and Schmidhuber (1997) as an alternative method to learn sequential patterns. LSTM networks are one of classes of recurrent neural networks (RNNs), but it has the advantage to retaining information over a long time-span compared with RNNs (LeCun et al., 2015; Fischer and Krauss, 2018). Graves and Schmidhuber (2005) demonstrate that LSTM networks are able to overcome the previously inherent problems and memorize temporal patterns over a long period of time.

LSTM networks are comprised of an input layer, several hidden layers, and an output layer.

The most important characteristics of LSTMs is memory cells which contained in the hidden layers.

Fig. 1 illustrates the structure of an LSTM memory cell. As we can see, for each memory cell, $x_{t}$ and $h_{t}$ correspond to the input and hidden state respectively, at time $t$, and $i_{t}, o_{t}$ and $f_{t}$, are the gates which are called input, output and forget gates, respectively, $s_{t}$ is adjusting its cell state. It is worth noting that the input gate decides which data can be added into the memory cell, the output gate decides which data from the memory cell can be used as output, and the forget gate decides which data should be deleted from the memory cell. The calculations for each state and gate are performed as the following formulas. 

selection issue, which initiates the foundation of Modern Portfolio Theory (MPT). In this model,

Fig.1. Structure of LSTM memory cell following Fischer and Krauss (2018)

$f_{t}=\operatorname{sigmoid}\left(W_{f, x} x_{t}+W_{f, h} h_{t-1}+b_{f}\right)$

$\tilde{s}_{t}=\operatorname{sigmoid}\left(W_{\tilde{s}, x} x_{t}+W_{\tilde{s}, h} h_{t-1}+b_{\tilde{s}}\right)$

$i_{t}=\operatorname{sigmoid}\left(W_{i, x} x_{t}+W_{i, h} h_{t-1}+b_{i}\right)$

$s_{t}=f_{t} * s_{t-1}+i_{t} * \tilde{s}_{t}$

$o_{t}=\operatorname{sigmoid}\left(W_{o, x} x_{t}+W_{o, h} h_{t-1}+b_{o}\right)$

$h_{t}=o_{t} * \tanh \left(s_{t}\right)$

Where $W_{f, x}, W_{f, h}, W_{\tilde{s}, x}, W_{\tilde{s}, h}, W_{i, x}, W_{i, h}, W_{o, x}$ and $W_{o, h}$ are weight matrices, $b_{f}, b_{\tilde{s}}$, $b_{i}$, and $b_{o}$ are bias vectors of the respective gates. Those bias vectors are added to increase the flexibility of the model to fit the data. Bias vectors $b_{\tilde{s}}, b_{i}$, and $b_{o}$ are initialized to zero, but the bias $b_{f}$ for the forget gate in LSTM is initialized to 1.0 (Jozefowicz et al., 2015). The symbol of * indicates element-wise multiplication. Because of this selective process of information, LSTM is able to deal with longer temporal patterns.

\subsection{Mean-variance model}

Mean-variance (MV) model proposed by Markowitz (1952) in order to solve optimal portfolio investment return and risk are quantified by expected return and variance, respectively. Santos and

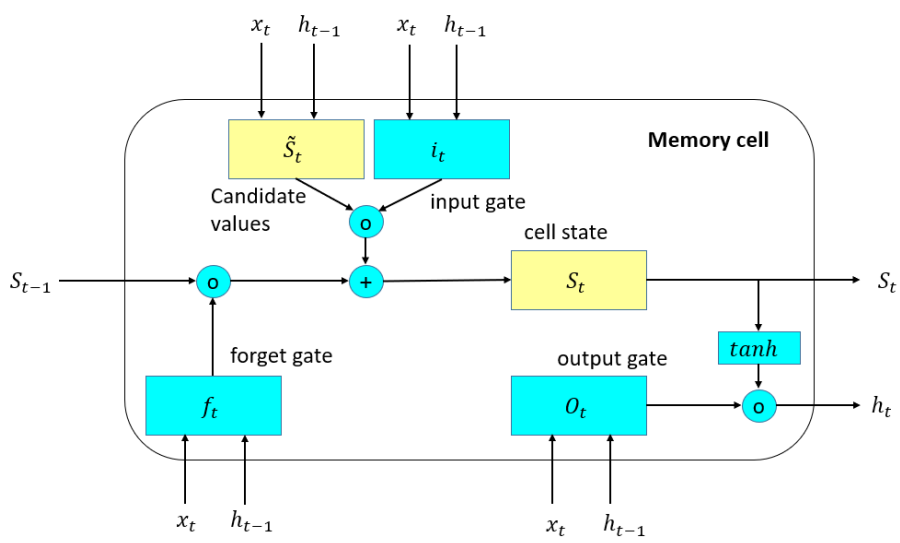


272 prefer the lower risk portfolios with constant expected returns or the higher expected return

273 portfolios with constant risk level. To solve this issue, a set of optimal solutions is generated, named

274 an efficient investment frontier. The model can be described by the following formulas:

$275 \quad \underset{w_{i}, \ldots, w_{n}}{\operatorname{Min}} \sum_{i=1}^{n} \sum_{j=1}^{n} w_{i} w_{j} \delta_{i j}$

$w_{i}, \ldots, w_{n} \sum_{i=1}^{n} w_{i} \mu_{i}$

$277 \quad$ Subject to: $\left\{\begin{array}{c}\sum_{i=1}^{n} w_{i}=1 \\ 0 \leq w_{i} \leq 1, \forall i=1, \ldots, n\end{array}\right.$

Where $w_{i}$ and $w_{j}$ represent the initial value invested in the portfolio or asset $i$ and asset $j$.

$\delta_{i j}$ specifies covariance between assets $i$ and asset $j . \mu_{i}$ is expected return on asset $i$. Following

Paiva et al. (2019), a variable $\lambda$ called risk aversion coefficient is integrated into the model to

$\underset{w_{i}, \ldots, w_{n}}{\operatorname{Min}} \lambda\left[\sum_{i=1}^{n} \sum_{j=1}^{n} w_{i} w_{j} \delta_{i j}\right]-(1-\lambda)\left[\sum_{i=1}^{n} w_{i} \mu_{i}\right]$

$$
\text { Subject to: }\left\{\begin{array}{c}
\sum_{i=1}^{n} w_{i}=1 \\
0 \leq w_{i} \leq 1, \forall i=1, \ldots, n
\end{array}\right.
$$

As a result, a group of optimal portfolios constitute an effective frontier can be derived and

introduced to the investor. So, the investor could select the point among these possible solutions according to his or her risk preference.

\subsection{Proposed model: LSTM+MV}

Many researches always ignore the fact that the purpose of forecasting financial market is not to show off the accuracy of a model but to apply these good results into the real market so as to 
process, high-quality asset input would be very helpful for the optimal portfolio formation. Given the important role that MV method plays in portfolio management area, we will continue to adopt this classical model, moreover, we will study deeply the preliminary selection of assets in order to provide MV model with better asset inputs. In this regard, this study puts forward a mixed method named LSTM+MV combining the advantages of deep learning LSTM method in time-series forecasting with the effectiveness of MV model in portfolio optimisation, aiming to improve the investment decision-making process.

There are two stages in our proposed model. In the first stage, LSTM method is applied to predict the return of the sample stocks in the next period. All the predicted results will be sorted in descending order and the top stocks will enter into the next phase. In the second stage, the Markowitz's MV model will be used to obtain the capital allocation proportion for each stock that has been entered.

\subsubsection{Input variable selection}

The selection of input variables is extremely necessary for time-series prediction tasks. In the light of previous literatures, technical indicators are effective features to describe and reflect the real market situation. For instance, Chen and Hao (2018) suggest that Exponential Moving Average (EMA), Relative Strength Index (RSI) and Momentum Index (MoM) are correlated with changes in stock market. Kara et al (2011) select ten technical indicators as input feature subsets. Also, financial time-series forecasting is always explained by the lagged observations. For example,

311 Fischer and Krauss (2018) use a return time sequence length of 240 for training. Paiva et al. (2019) use several lagged variables of return as inputs to predict the future return of stocks. Hereby, after referring to the views of domain papers, we make feature selection by recursive feature elimination 
314 (RFE). To be specific, RFE works by recursively removing features and building a model on those

315 features that remain. It uses the model accuracy to identify which features contribute the most to

316 predicting the target feature (return in $t+1$ period). We use RFE with the logistic regression

317 algorithm to select the features with a ratio greater than 0.3. Fig. 2 shows the results of feature

318 selection using RFE. We finally choose twenty important indicators as input variables, including

319 five technical indicators and fifteen lagged observations about return. The values of all technical

320 indicators are standardized in the range of $(-1,+1)$, in order to avoid the errors caused by different

321 indicators of different numerical ranges. Table 2 summarises the selected input variables. Among

322 the variables are return measures based on open, close, high, low prices, and volume. A brief explanation of each indicator is as following.

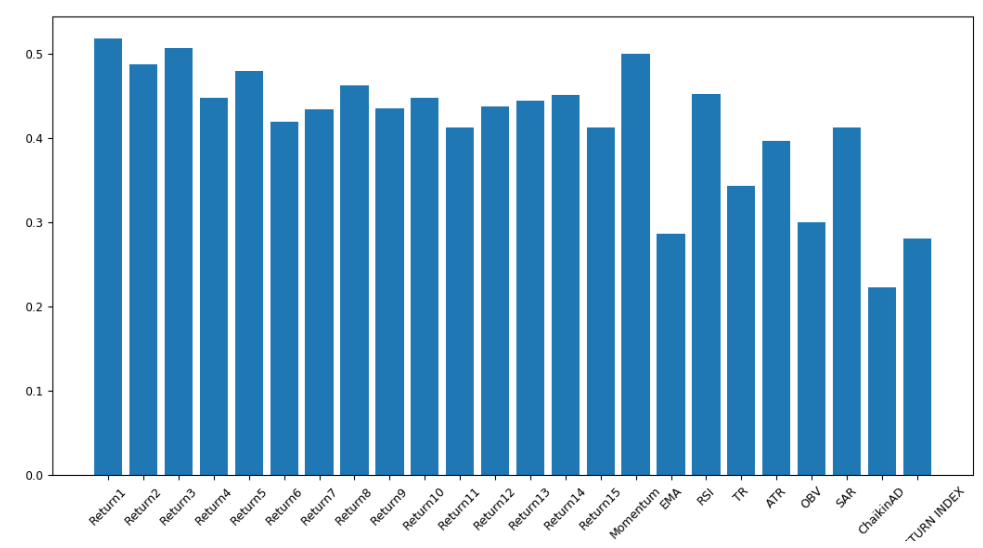

Fig. 2. Feature selection results

Table 2 Input features summary

\begin{tabular}{|c|c|c|c|}
\hline Attribute & Details & Attribute & Details \\
\hline 1 & $r_{1}=\ln \left(\frac{\text { close }_{\text {price }}}{\text { close }_{\text {price }}}\right)$ & 11 & 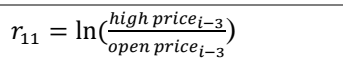 \\
\hline 2 & $r_{2}=\ln \left(\frac{\text { close price }_{i-1}}{\text { close price }}\right)$ & 12 & $r_{12}=\ln \left(\frac{\text { low price }}{\text { open price }}\right)$ \\
\hline 3 & $r_{3}=\ln \left(\frac{\text { close price }_{i-2}}{\text { close price } i-3}\right)$ & 13 & $r_{13}=\ln \left(\frac{\text { low price }_{i-1}}{\text { open price }_{i-1}}\right)$ \\
\hline 4 & $r_{4}=\ln \left(\frac{\text { close }^{\text {price }}}{\text { close }_{i-3} \text { price }_{i-4}}\right)$ & 14 & 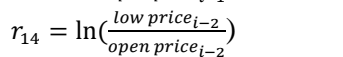 \\
\hline 5 & $r_{5}=\ln \left(\frac{\text { high price }}{\text { open price }_{i}}\right)$ & 15 & 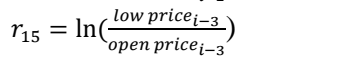 \\
\hline 6 & 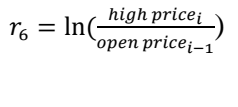 & 16 & $\begin{array}{l}\text { Relative Strength Index } \\
\text { (close price, period }=14 \text { ) }\end{array}$ \\
\hline 7 & 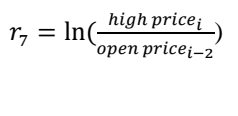 & 17 & $\begin{array}{l}\text { Momentum Index (close } \\
\text { price, period }=10 \text { ) }\end{array}$ \\
\hline 8 & 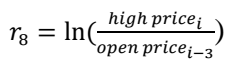 & 18 & True range (high, low, and \\
\hline
\end{tabular}




\begin{tabular}{|c|c|c|c|}
\hline & & & close price) \\
\hline 9 & 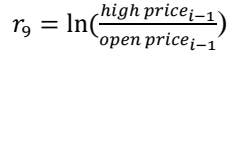 & 19 & $\begin{array}{l}\text { Average true range (high, } \\
\text { low and close price, } \\
\text { period }=14) \text { ) }\end{array}$ \\
\hline 10 & $r_{10}=\ln \left(\frac{\text { high price }_{i-2}}{\text { open price }}\right.$ pr-2 $\left._{i-2}\right)$ & 20 & $\begin{array}{l}\text { Parabolic SAR (high and } \\
\text { low price, acceleration }= \\
0.02, \text { maximum }=0 \text { ) }\end{array}$ \\
\hline
\end{tabular}

1) Simple return

Set $P_{t}^{i}$ as the price process of stock $i$ at time $t$, with $i \epsilon\{1,2 \ldots, n\}$ and $R_{t}^{m, i}$ as the simple

331 return for a stock $i$ over $t$ periods, i.e., $R_{t}^{m, i}=\frac{P_{t}^{i}}{P_{t-m}^{i}}$.

2) Relative Strength Index (RSI)

RSI, a momentum indicator, is able to measure the magnitude of the rise and fall in prices

recently. It is very effective in assessing the overbought/oversold condition of an asset. According

to the parameters of this indicator in existing researches (Paiva et al., 2019; Chen and Hao, 2018;

Patel et al., 2015), this paper set the period as 14 .

4) True range (TR)

TR is the maximum change in the price of the day compared to the previous day.

ATR is a technical analysis indicator that reflects market volatility through decomposing the entire range of an asset price for a period. 
it will remind us when the direction of the price changes, in another words, it will adjust as prices change so as to attract investors' attention.

\subsubsection{Generation of training and testing sets} period", involving a training period of 750 days and a testing period of 250 days (Fischer and areas together form our "study period", 1000 days.

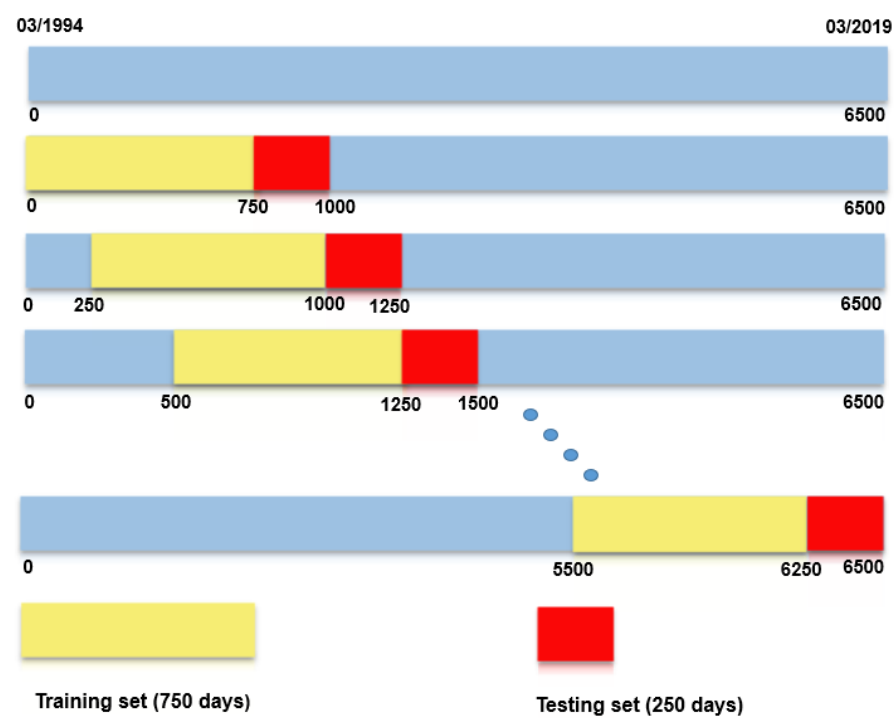

Fig. 3. overlapping training-testing sets 


\subsubsection{Process of optimal portfolio formation}

The proposed model LSTM+MV in this paper is on the basis of technical analysis as well as the historical asset prices identification. On this account, we follow the assumption of Fama (1965) who holds the view that history behaviour trend of the price change in individual assets are inclined to repeat in the future. The primary objective of the LSTM method here is to forecast the relative return rate of each stock in $t+1$ trading day on the basis of information before time $t$. In LSTM networks of our proposed model, some sequences of input features are required for training, that is, the values of input features at points in consecutive time. With regards to the training of the LSTM networks, three advanced methods are applied through Keras. First, Adam (Kingma and Ba, 2014) is used as the optimiser to improve the neural network. This selection is inspired from some existing researches (Kingma and Ba, 2014; Reimers and Gurevych; 2017; Kraus and Feuerriegel, 2017), as they testify that Adam is appropriate for deep LSTM networks and has a better performance in optimising the neural network. Second, referring to Srivastava et al. (2014), we make use of dropout regularization technique on the hidden layer. In this case, randomly selected neurons are dropped

377 during training times, along with corresponding input and output connections, which is able to reduce overfitting efficiently (Srivastava et al., 2014; Fischer and Krauss, 2018). In the case of 379 Adam optimiser, we also carry an initial experiment using a part of the sample, the result shows that the model performance decreases as the dropout rate increases, hence, we set the dropout rate relatively low as 0.1 . Third, we perform random search method to dynamically find a good combination of hyperparameters based on the above settings. Many empirical evidences have shown the effectiveness of random search in optimising the parameters (Bergstra and Bengio, 2012; 

activation function; (4) the number of neurons per hidden layer, ranging from 2 to 200. Finally, the specified topology of the LSTM network is confirmed. We set 20 features and 72 timesteps in input layer. And in LSTM layer, we set 60 hidden neurons and 0.1 for dropout rate. In dense layer, we apply 16 neurons and relu activation function. Also, we set one neuron and sigmoid activation function in output layer, which is a standard configuration (Fischer and Krauss, 2018). Since the optimal sequence length is 72 , approximately covering the data of three testing months. Thus, overlapping sequences of 72 consecutives are generated. In total, 22 study period contain about 429,000 of those sequences, in which approximately 321,750 are utilized for in-sample training, and 107,250 are utilized for out-of-sample predictions. For each study period, there are about 19,500 of those sequences. Suppose that we would like to find whether an asset has the potential to reach higher return in $t+1$. Then, we will collect all data of that asset before the trading session at $t_{0}$ in order to achieve this goal. According to LSTM principles, the data series from previous days would be put into the model to implement experiment.

Once all the assets are predicted, one by one, we will rank all stocks for each period $t+1$ in descending order of this predicted return. Only the top $k$ of the ranking with the higher return assets that are considered to qualify to enter into the next phase. The purpose of the second stage is to obtain the capital allocation proportion for each asset. And the Markowitz's MV model will be used to carry on this stage. It is worth clarifying that the proposed model does not take into account investors' risk preference and risk-free assets, thus, the portfolios exclusively compose of risky assets. According to the way of Malkiel (2007) letting a blindfolded monkey throw darts at a 
portfolios. From a statistical perspective, 50,000 random portfolios basically cover most possible

408

409

410

411 allocated to the portfolio with the lowest variance. As such, when the assets and the respective investment proportions are confirmed, the next step is to allocate capital at the opening of the next trading day. We will go long the top $k$ assets during the investment day. The detailed process of the proposed method is shown in Fig. 4.

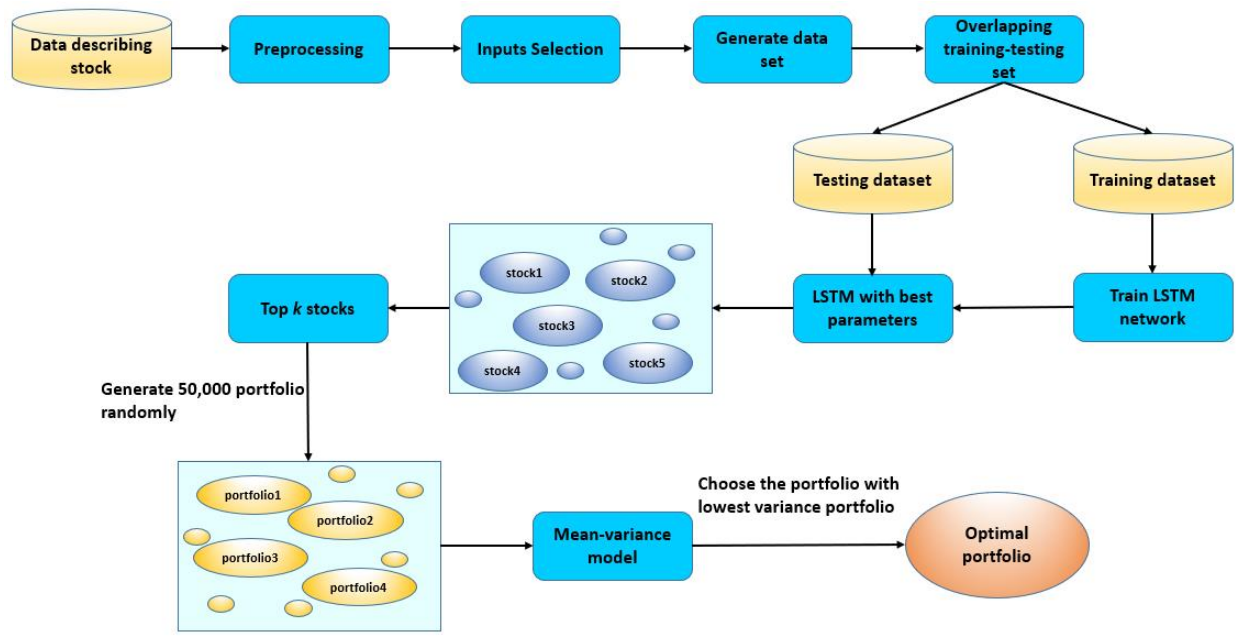

Fig. 4. The scheme of proposed model

\subsubsection{Benchmark models for prediction: SVM, RAF, DNN and ARIMA}

In order to benchmark the LSTM, three representative machine learning models, support vector machine, random forest, deep neural network, as well as a traditional statistical model named Autoregressive Integrated Moving Average that is often applied for time-series prediction. We will introduce the principles of each model in the following paragraphs.

Support Vector Machine: This technique aims to solve issues related to classification, 

in order to estimate the dependent variable value (Loureiro et al., 2018). needs tuning (Fischer and Krauss, 2018). layer. Dropout is set to 0.2 .

fitted to predict time-series data in future, and ARIMAX extends ARIMA model by including exogenous variables (Pektas and Cigizoglu, 2013). This paper uses ARIMAX model as one of baseline models.

\subsubsection{Baseline strategies for portfolio formation}

In reality, except for MV model, equal-weighted portfolio and Black-Litterman (BL) model

442 are also popular. It is worth noting that we originally used the BL model as one of the baselines, but in the end, we found that we could not get a prominent and consistent result to explain. Maybe the parameters of different models need to be adjusted or due to some other reasons we have not 
strategies are based on the LSTM+MV model proposed in the prior section and used to compare

with this model's changes and performance.

\section{(1) Alternative model: Machine learning + MV}

This kind of model's design is similar with the logical structure of the LSTM+MV model. The

main objective is to find out whether different prediction results of asset return will have an impact

predicted by one machine learning method with better forecasting performance in the in the first

The second stage, portfolio optimisation, applying the Markowitz's MV method is maintained.

(2) Alternative model: Machine learning $+1 / \mathrm{N}$ predict assets returns in $t+1$, and then rank these assets according to the predicted results. Finally, the top $k$ assets will enter into the second stage and receive the same proportion of investment.

Notice that $k$ should be consistent with the number defined in the LSTM+MV model.

\section{(3) Alternative model: Random + MV or $1 / \mathrm{N}$}

This kind of baseline strategy differs from the previous baselines in terms of the asset predictions, but the number of assets should be same as the number defined by the other models. 
469 objective of this kind of baseline strategy is to examine the necessity of asset preselection using

470 machine learning.

471 4. Experiments and Results

4724.1 Results analysis in the first stage: prediction

473 In this section, we use five criterions to evaluate predictive accuracy, mean square error (MSE),

474 root-mean-square error (RMSE), mean absolute percentage error (MAPE), mean absolute error

475 (MAE) and coefficient of determination $\left(R^{2}\right)$. Tables 3 to 5 summarise the best results achieved for

476 each model applied according to the different evaluation metrics employed. As can be seen from

477 three tables, the majority of indicators corresponding to LSTM model perform better than the index

478 value of other models, but several exceptions also exist. For example, the MAE and MAPE

479 indicator of stock SSE where the prediction result of LSTM is larger than that of SVM and DNN

480 respectively. Another example is that the $R^{2}$ of 3 stocks (BP, JM, SG) predicting by SVM are

481 higher than that of LSTM, and the $R^{2}$ of 2 stocks (BAT, PEA) using RAF are higher than that of

482 LSTM too.

483

Table 3 Comparison of prediction performance

\begin{tabular}{l|lllll|lllll}
\hline & \multicolumn{5}{|c|}{ LSTM } & \multicolumn{5}{c}{ SVM } \\
\hline Stock & MSE & RMSE & MAPE & MAE & $\mathrm{R}^{2}$ & MSE & RMSE & MAPE & MAE & $\mathrm{R}^{2}$ \\
\hline TES & 0.0031 & 0.0557 & 67.99 & 0.0364 & 0.4209 & 0.0042 & 0.0651 & 167.92 & 0.0427 & 0.3108 \\
AST & 0.0032 & 0.0568 & 165.53 & 0.0324 & 0.2806 & 0.0089 & 0.0942 & 190.68 & 0.0512 & 0.1856 \\
BAR & 0.0007 & 0.0265 & 6.63 & 0.0159 & 0.2631 & 0.0050 & 0.0608 & 22.47 & 0.0335 & 0.1200 \\
BP & 0.0053 & 0.0727 & 123.00 & 0.0413 & 0.1121 & 0.0054 & 0.0732 & 114.88 & 0.0450 & 0.1379 \\
BAT & 0.0019 & 0.0439 & 7.76 & 0.0296 & 0.1259 & 0.0064 & 0.0731 & 25.50 & 0.0404 & 0.0862 \\
HAL & 0.0050 & 0.0709 & 266.49 & 0.0378 & 0.2288 & 0.0054 & 0.0735 & 221.27 & 0.0397 & 0.1359 \\
HH & 0.0015 & 0.0390 & 25.10 & 0.0214 & 0.2395 & 0.0024 & 0.0484 & 55.71 & 0.0290 & 0.1349 \\
JM & 0.0063 & 0.0797 & 221.26 & 0.0495 & 0.1327 & 0.0099 & 0.0997 & 226.03 & 0.0431 & 0.1718 \\
LG & 0.0009 & 0.0293 & 17.42 & 0.0155 & 0.1585 & 0.0010 & 0.0317 & 18.71 & 0.0176 & 0.1210 \\
MSG & 0.0029 & 0.0540 & 17.06 & 0.0301 & 0.2630 & 0.0040 & 0.0629 & 19.62 & 0.0390 & 0.2141 \\
PEA & 0.0018 & 0.0426 & 9.06 & 0.0227 & 0.1100 & 0.0026 & 0.0513 & 10.95 & 0.0323 & 0.1816 \\
REL & 0.0028 & 0.0532 & 35.08 & 0.0267 & 0.1557 & 0.0029 & 0.0539 & 37.78 & 0.0297 & 0.1145 \\
\hline
\end{tabular}




\begin{tabular}{l|lllll|lllll}
\hline RB & 0.0002 & 0.0146 & 1.98 & 0.0094 & 0.4578 & 0.0003 & 0.0162 & 2.22 & 0.0104 & 0.3768 \\
RDSB & 0.0068 & 0.0825 & 158.40 & 0.0417 & 0.2960 & 0.0062 & 0.0785 & 154.90 & 0.0417 & 0.1093 \\
SG & 0.0039 & 0.0622 & 74.90 & 0.0302 & 0.2545 & 0.0047 & 0.0684 & 71.50 & 0.0367 & 0.3592 \\
SJ & 0.0028 & 0.0525 & 16.29 & 0.0258 & 0.6139 & 0.0031 & 0.0552 & 17.00 & 0.0279 & 0.1218 \\
SCH & 0.0011 & 0.0329 & 265.81 & 0.0189 & 0.1638 & 0.0014 & 0.0368 & 232.70 & 0.0224 & 0.1553 \\
ST & 0.0046 & 0.0680 & 43.34 & 0.0388 & 0.1441 & 0.0070 & 0.0837 & 77.83 & 0.0450 & 0.1454 \\
SG & 0.0052 & 0.0726 & 61.96 & 0.0394 & 0.2809 & 0.0077 & 0.0876 & 94.77 & 0.0510 & 0.2001 \\
SSE & 0.0041 & 0.0642 & 196.59 & 0.0346 & 0.4572 & 0.0042 & 0.0645 & 245.40 & 0.0335 & 0.2977 \\
VG & 0.0045 & 0.0672 & 138.70 & 0.0385 & 0.3448 & 0.0071 & 0.0844 & 237.70 & 0.0469 & 0.2810 \\
\hline
\end{tabular}

Table 4 Comparison of prediction performance

\begin{tabular}{l|lllll|llllll}
\hline & \multicolumn{7}{|c|}{ DNN } & \multicolumn{7}{c}{ RAF } \\
\hline Stock & MSE & RMSE & MAPE & MAE & $\mathrm{R}^{2}$ & MSE & RMSE & MAPE & MAE & MSE & $\mathrm{R}^{2}$ \\
\hline TES & 0.0130 & 0.1160 & 317.26 & 0.0590 & 0.1300 & 0.0058 & 0.0758 & 189.56 & 0.0526 & 0.0058 & 0.3593 \\
AST & 0.0100 & 0.1010 & 194.48 & 0.0480 & 0.1410 & 0.0046 & 0.0676 & 162.97 & 0.0409 & 0.0046 & 0.2119 \\
BAR & 0.0020 & 0.0450 & 10.04 & 0.0220 & 0.0800 & 0.0056 & 0.0643 & 23.25 & 0.0370 & 0.0056 & 0.1563 \\
BP & 0.0150 & 0.1210 & 484.08 & 0.0590 & 0.1020 & 0.0213 & 0.1402 & 653.1 & 0.0817 & 0.0213 & 0.1261 \\
BAT & 0.0040 & 0.0630 & 10.44 & 0.0340 & 0.1770 & 0.0025 & 0.0495 & 8.87 & 0.0285 & 0.0025 & 0.2855 \\
HAL & 0.0080 & 0.0900 & 273.28 & 0.0460 & 0.1120 & 0.0062 & 0.0787 & 234.59 & 0.0413 & 0.0062 & 0.2100 \\
HH & 0.0060 & 0.0750 & 89.25 & 0.0380 & 0.1340 & 0.0069 & 0.0832 & 49.11 & 0.0472 & 0.0069 & 0.2047 \\
JM & 0.0150 & 0.1220 & 495.23 & 0.0660 & 0.1210 & 0.0256 & 0.1503 & 296.76 & 0.0847 & 0.0256 & 0.1026 \\
LG & 0.0030 & 0.0540 & 34.60 & 0.0230 & 0.6890 & 0.0023 & 0.0475 & 24.14 & 0.0282 & 0.0023 & 0.3095 \\
MSG & 0.0060 & 0.0760 & 35.77 & 0.0390 & 0.1630 & 0.0045 & 0.0674 & 31.37 & 0.0362 & 0.0045 & 0.2057 \\
PEA & 0.0060 & 0.0780 & 17.88 & 0.0340 & 0.0360 & 0.0020 & 0.0442 & 9.69 & 0.0265 & 0.0020 & 0.2403 \\
REL & 0.0047 & 0.0684 & 53.22 & 0.0407 & 0.1130 & 0.0046 & 0.0678 & 43.69 & 0.0404 & 0.0046 & 0.1153 \\
RB & 0.0010 & 0.0300 & 3.71 & 0.0150 & 0.1530 & 0.0002 & 0.0140 & 1.91 & 0.0085 & 0.0002 & 0.3735 \\
RDSB & 0.0170 & 0.1310 & 221.60 & 0.0670 & 0.1030 & 0.0095 & 0.0972 & 155.13 & 0.0666 & 0.0095 & 0.1051 \\
SG & 0.0060 & 0.0790 & 95.74 & 0.0380 & 0.1293 & 0.0045 & 0.0672 & 81.20 & 0.0470 & 0.0045 & 0.1064 \\
SJ & 0.0090 & 0.0950 & 38.24 & 0.0450 & 0.1407 & 0.0037 & 0.0607 & 19.67 & 0.0363 & 0.0037 & 0.2391 \\
SCH & 0.0030 & 0.0550 & 270.87 & 0.0260 & 0.0840 & 0.0014 & 0.0368 & 291.32 & 0.0244 & 0.0014 & 0.1528 \\
ST & 0.0090 & 0.0930 & 122.99 & 0.0500 & 0.0826 & 0.0064 & 0.0797 & 48.82 & 0.0474 & 0.0064 & 0.1515 \\
SG & 0.0120 & 0.1080 & 196.68 & 0.0600 & 0.1973 & 0.0062 & 0.0784 & 64.88 & 0.0460 & 0.0062 & 0.1032 \\
SSE & 0.0090 & 0.0940 & 191.07 & 0.0460 & 0.2891 & 0.0050 & 0.0703 & 312.05 & 0.0407 & 0.0050 & 0.1439 \\
VG & 0.0090 & 0.0950 & 236.85 & 0.0490 & 0.0100 & 0.0060 & 0.0771 & 186.13 & 0.0450 & 0.0060 & 0.2093 \\
\hline
\end{tabular}

Table 5 Comparison of prediction performance

\begin{tabular}{l|lllll}
\hline & \multicolumn{5}{|c}{ ARIMA } \\
\hline Stock & MSE & RMSE & MAPE & MAE & $\mathrm{R}^{2}$ \\
\hline TES & 32.22 & 5.68 & 8618.9 & 5.89 & 0.2427 \\
AST & 171.13 & 13.08 & 1222.3 & 10.37 & 0.1717 \\
BAR & 7.90 & 2.81 & 389.45 & 2.56 & 0.1399 \\
BP & 14.83 & 3.85 & 7780.1 & 3.94 & 0.1038 \\
BAT & 1.67 & 1.29 & 200.90 & 0.80 & 0.0444 \\
HAL & 50.50 & 7.11 & 19703 & 5.79 & 0.0805 \\
\hline
\end{tabular}




\begin{tabular}{l|lllll}
\hline HH & 3.89 & 1.97 & 888.75 & 1.36 & 0.0772 \\
JM & 2.63 & 1.62 & 2701.7 & 1.05 & 0.0176 \\
LG & 3.08 & 1.75 & 518.96 & 1.53 & 0.1103 \\
MSG & 8.75 & 2.96 & 639.18 & 1.72 & 0.1648 \\
PEA & 7.25 & 2.69 & 416.42 & 2.43 & 0.1239 \\
REL & 67.52 & 2.60 & 1065.3 & 2.14 & 0.1534 \\
RB & 2.25 & 1.50 & 214.61 & 1.56 & 0.2945 \\
RDSB & 11.52 & 3.39 & 2297.2 & 1.99 & 0.0678 \\
SG & 3.50 & 1.87 & 884.67 & 1.66 & 0.0570 \\
SJ & 11.70 & 3.42 & 795.99 & 3.54 & 0.1286 \\
SCH & 39.27 & 6.27 & 34652 & 5.74 & 0.1363 \\
ST & 40.70 & 6.38 & 3414.5 & 6.49 & 0.3774 \\
SG & 51.26 & 7.16 & 5028.2 & 4.07 & 0.2443 \\
SSE & 2.66 & 1.63 & 6030.1 & 1.53 & 0.0299 \\
VG & 1.26 & 1.12 & 3880.9 & 0.86 & 0.0137 \\
\hline
\end{tabular}

Mean square error (MSE): It is an indicator measuring the average squared difference between

489 the observed values and predicted values. From Table 3, Table 4 and Table 5, we find the following 490 average MSE result: 0.0033 for LSTM model, 0.0047 for SVM model, 0.008 for DNN model, 0.64 491 for RAF model and 25.49 for ARIMA model.

Root-mean-square error (RMSE): It is another effective indicator measuring differences 493 between the observed values and predicted values. As can be seen from Tables 3 to 5, LSTM 494 exhibits favourable mean RMSE 0.0543, followed by SVM (0.0649), then the indicator for DNN and RAF equals to 0.0852 and 0.0723 , but 3.817 for ARIMA model.

Mean absolute percentage error (MAPE): It measures the prediction deviation proportion in 497 terms of the true value. After comparing different models in terms of MAPE, we can get the average results: 91.44 for LSTM model, 106.93 for SVM model, 161.58 for DNN model, 137.53 for RAF model and 2560 for ARIMA model. model, 0.0432 for RAF model and 3.1913 for ARIMA model. 
The $R^{2}$ of RAF, SVM and DNN is a little higher than that of LSTM in terms of several stocks,

505 but on average, the LSTM model has the highest $R^{2}$ of 0.2621 , followed by RAF (0.1958) and

LSTM ranges from 0.1100 to 0.6139 , similar to several existing financial researches (Gatev et al.,

2006; Fischer and Krauss, 2018). To be specific, the main purpose of the preselection phase is to forecast the return of assets and select assets with higher potential returns. Unlike researches on explanatory modelling aiming to explain causal relationships and the importance of each indicator,

511 predictive modelling is primarily concerned with accuracy and error in order to predict future observations (Shmueli, 2010; Gandhmal and Kumar, 2019). In this case, the effectiveness of this kind of model is primarily determined by accuracy measures, such as RMSE and MSE, rather than the value of $R^{2}$ (Alexander et al., 2015; Gandhmal and Kumar, 2019). regarded as popular performance metrics since they can clearly present the average model

517 prediction error (Kao et al., 2013; Weng et al., 2018; Gandhmal and Kumar, 2019). For several other works, it is difficult to evaluate these metrics through direct comparison due to the difference of datasets. But we can compare the results with widely used methods in related researches. From Tables 3 to 5, the average values of MSE, RMSE, MAE for LSTM model are 0.0033, 0.0543 and 0.0303 respectively, which have showed superior performance in forecasting stock returns against existing works (Ticknor, 2013; Sadaei et al., 2016; Weng et al., 2018; Gandhmal and Kumar, 2019). 

worst. For example, for stock TES, the MSE in ARIMA equals to 32.21, which is 5000 times bigger

527 than MSE (0.0031) in LSTM.

\subsection{Results analysis in the second stage: optimal portfolio formation}

\subsubsection{Determination of the portfolio size}

530 Firstly, we analyse the characteristics of portfolios consisting of $k$ assets. Most of researches corresponding to portfolio formation for individual investors focus on only fewer than 10 assets (Kocuk and Cornuéjols, 2018; Tanaka et al., 2000; Almahdi and Yang, 2017), because holding too many different stocks is hard for an individual investor to manage. Ranguelova (2001) indicate that individual investors usually hold three or four stocks in their account on average. Paiva et al., (2019) discover that the portfolio with seven assets performs better than others with different numbers of assets. Hereby, assuming an individual investor holding less than or equal to 10 assets is realistic. Based on the above discussion, we choose $k \in\{4,5,6,7,8,9,10\}$, and then compare the performance of the model LSTM+MV with the other baseline strategies according to the dimensions annualised standard deviation, annualised mean return, annualised Sharpe ratio, and

540 Sortino ratio before transaction costs.

As can be seen from Fig. 5, there are four subgraphs. Specifically, the Y-axis of four sub-

544 From Fig. 5, it is clear that irrespective of the portfolio size $k$, the LSTM+MV shows greater

545 performance than the other strategies in three dimensions of annualised mean return, Sharpe ratio 
549 achieves the highest mean returns per year. In regard to annualised standard deviation, a risk metric,

550 differences among models are not obvious, the LSTM +MV is on a similar level as the other models,

551 with slightly higher values for $k=6,7,8$, thus we could not distinguish which models are good

552 or bad on this metric easily. In this study, we set risk-free ratio as 0.0125 , according to the British

553 treasuring bill rate in recent 10 years. With respect to Sharpe ratio, return per unit of risk, is highest

554 for the LSTM+MV. For example, when $k=9$, Sharpe ratio before transaction cost is 0.58 ,

555 compared to 0.40 for the LSTM+1/N, 0.46 for the SVM+MV, 0.36 for the $S V M+1 / N, 0.38$ for the

$556 \mathrm{RAF}+\mathrm{MV}, 0.28$ for $\mathrm{RAF}+1 / \mathrm{N}$. Sortino ratio, measuring the risk-adjusted return of an investment

557 portfolio. A clear advantage of the LSTM+MV can be seen for portfolios of each size. From the

558 perspective of different portfolio sizes, it is easy to find that the four indicators perform better overall in each model when $k=10$ than other sizes. Specifically, in model LSTM+MV, the portfolio with $k=10$ not only has a high mean return 0.136 , Sharpe ratio 0.58 and Sortino ratio 13.7, but also has a lower standard deviation 0.21 . And the same is true for the analysis of other models. From the above analysis, we focus the portfolio with $k=10$ in our subsequent analyses, which is also consistent with the research of Fischer and Krauss (2018). 

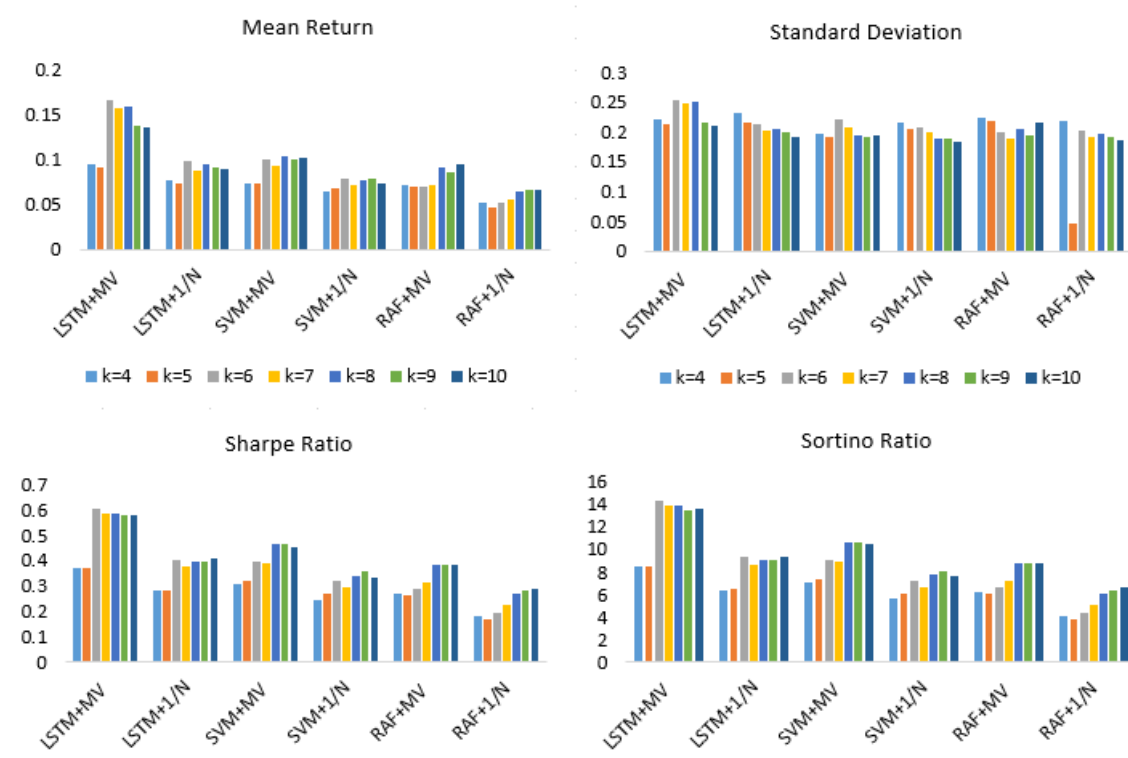

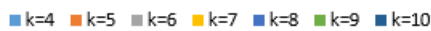

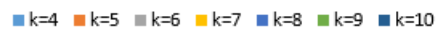

Fig. 5. Annualised performance characteristics for portfolios of different sizes

568

569

570

571

572

573 Guerard Jr et al., 2015; Paiva et al., 2019), we decide to simulate transaction costs as 0.10 bps,

5740.05 bps to present the results finally. Tables 6 to 8 provide insights of the financial performance

575 of the LSTM+MV, compared to the baselines, without transaction cost, including transaction cost

576 (0.1bps, 0.05bps) separately. Hence, Panel A, B and C depict daily return characteristics, daily risk

577 characteristics and annualised risk-return metrics respectively.

578

Table 6 Performance characteristics for portfolios without transaction cost

\begin{tabular}{|c|c|c|c|c|c|c|c|}
\hline & & LSTM+MV & $\mathrm{LSTM}+1 / \mathrm{N}$ & $\mathrm{SVM}+\mathrm{MV}$ & $\mathrm{SVM}+1 / \mathrm{N}$ & $\mathrm{RAF}+\mathrm{MV}$ & $\mathrm{RAF}+1 / \mathrm{N}$ \\
\hline \multirow[t]{4}{*}{ A } & Mean return & 0.0005 & 0.0004 & 0.0004 & 0.0003 & 0.0004 & 0.0003 \\
\hline & Standard deviation & 0.0134 & 0.0121 & 0.0124 & 0.0116 & 0.0137 & 0.0118 \\
\hline & Maximum & 0.1003 & 0.1100 & 0.0953 & 0.0965 & 0.1241 & 0.1052 \\
\hline & Minimum & -0.0748 & -0.0953 & -0.0744 & -0.1014 & -0.0749 & -0.1005 \\
\hline \multirow[t]{2}{*}{$\mathrm{B}$} & 1-percent VaR & 0.0330 & 0.0337 & 0.0336 & 0.0327 & 0.0385 & 0.0328 \\
\hline & 1-percent CVaR & 0.0439 & 0.0446 & 0.0451 & 0.0427 & 0.0514 & 0.0443 \\
\hline
\end{tabular}




\begin{tabular}{lllllll}
\hline 5-percent VaR & 0.0207 & 0.0189 & 0.0188 & 0.0178 & 0.0207 & 0.0188 \\
5-percent CVaR & 0.0306 & 0.0282 & 0.0285 & 0.0271 & 0.0318 & 0.0277 \\
Maximum drawdown & 2.5277 & 2.4182 & 2.9685 & 2.2441 & 2.5612 & 2.1990 \\
C Mean return & 0.1367 & 0.0913 & 0.1022 & 0.0743 & 0.0963 & 0.0676 \\
Standard deviation & 0.2125 & 0.1919 & 0.1963 & 0.1844 & 0.2176 & 0.1878 \\
Sharpe ratio & 0.5845 & 0.4105 & 0.4569 & 0.3354 & 0.3852 & 0.2932 \\
Sortino ratio & 13.7078 & 9.3844 & 10.4918 & 7.6352 & 8.8549 & 6.6693 \\
\hline
\end{tabular}

579

580

Table 7 Performance characteristics for portfolios including transaction cost $(0.05 \mathrm{bps})$

\begin{tabular}{lllllll}
\hline & LSTM+MV & LSTM+1/N & SVM+MV & SVM+1/N & RAF+MV & RAF+1/N \\
\hline A Mean return & 0.0003 & 0.0003 & 0.0003 & 0.0003 & 0.0003 & 0.0003 \\
Standard deviation & 0.0125 & 0.0139 & 0.0155 & 0.0125 & 0.0139 & 0.0134 \\
Maximum & 0.1152 & 0.1397 & 0.1535 & 0.1031 & 0.1186 & 0.1302 \\
Minimum & -0.1027 & -0.1746 & -0.2575 & -0.1322 & -0.1387 & -0.1698 \\
B 1-percent VaR & 0.0341 & 0.0368 & 0.0408 & 0.0337 & 0.0384 & 0.0365 \\
1-percent CVaR & 0.0472 & 0.2119 & 0.0598 & 0.1897 & 0.0529 & 0.2047 \\
5-percent VaR & 0.0196 & 0.0210 & 0.0232 & 0.0196 & 0.0209 & 0.0202 \\
5-percent CVaR & 0.0293 & 0.0424 & 0.0352 & 0.0379 & 0.0321 & 0.0409 \\
Maximum drawdown & 2.3442 & 2.5068 & 2.7753 & 2.9920 & 3.6550 & 2.3043 \\
C Mean return & 0.0765 & 0.0789 & 0.0792 & 0.0780 & 0.0691 & 0.0630 \\
Standard deviation & 0.1988 & 0.2203 & 0.2462 & 0.1988 & 0.2207 & 0.2129 \\
Sharpe ratio & 0.3218 & 0.2978 & 0.2710 & 0.3294 & 0.2567 & 0.2374 \\
Sortino ratio & 0.0906 & 0.0834 & 0.0753 & 0.0926 & 0.0720 & 0.0667 \\
\hline
\end{tabular}

581

582

Table 8 Performance characteristics for portfolios including transaction cost $(0.1 \mathrm{bps})$

\begin{tabular}{lllllll}
\hline & LSTM+MV & LSTM+1/N & SVM+MV & SVM+1/N & RAF+MV & RAF+1/N \\
\hline A Mean return & 0.0003 & 0.0003 & 0.0003 & 0.0003 & 0.0002 & 0.0003 \\
Standard deviation & 0.0149 & 0.0140 & 0.0153 & 0.0126 & 0.0155 & 0.0135 \\
Maximum & 0.1524 & 0.1411 & 0.1445 & 0.1041 & 0.1658 & 0.1314 \\
Minimum & -0.2124 & -0.1762 & -0.2340 & -0.1334 & -0.2541 & -0.1714 \\
B 1-percent VaR & 0.0388 & 0.0372 & 0.0405 & 0.0340 & 0.0408 & 0.0368 \\
1-percent CVaR & 0.0582 & 0.0212 & 0.0600 & 0.1915 & 0.0612 & 0.2066 \\
5-percent VaR & 0.0222 & 0.2140 & 0.0228 & 0.0198 & 0.0229 & 0.0204 \\
5-percent CVaR & 0.0344 & 0.0428 & 0.0352 & 0.0383 & 0.0356 & 0.0413 \\
Maximum drawdown & 2.7861 & 2.5068 & 3.0653 & 2.9920 & 2.5326 & 2.3043 \\
C Mean return & 0.0763 & 0.0787 & 0.0705 & 0.0787 & 0.0616 & 0.0636 \\
Standard deviation & 0.2366 & 0.2224 & 0.2434 & 0.2007 & 0.2468 & 0.2149 \\
$\quad$ Sharpe ratio & 0.2697 & 0.2975 & 0.2384 & 0.3300 & 0.1990 & 0.2379 \\
Sortino ratio & 0.0752 & 0.0833 & 0.0662 & 0.0924 & 0.0553 & 0.0665 \\
\hline
\end{tabular}

Return characteristics: In panel A of Table 6, we can see that the LSTM+MV exhibits 
584 favourable daily mean return 0.0005 , and the SVM+MV has the lowest standard deviation as 585 0.0116. After including transaction cost $0.05 \mathrm{bps}$, in panel A of Table 6, we can find that all the 586 models have almost same daily return 0.0003 , the LSTM+MV model and SVM+1/N model have 587 the lowest standard deviation. After including transaction cost $0.1 \mathrm{bps}$, in panel A of Table 7, $588 \quad \mathrm{SVM}+1 / \mathrm{N}$ model has a better risk level, daily standard deviation equals to 0.0126 .

589 Risk characteristics: In panel B of Table 6, Table 7 and Table 8, we can see a mixed picture 590 corresponding to risk characteristics. Before transaction cost, $\mathrm{SVM}+1 / \mathrm{N}$ achieved the best place with a 1-percent VaR of 0.0327, 5-percent VaR of 0.0178, 1-percent CVaR of 0.0427 and 5-percent CVaR of 0.0271 . After including transaction cost 0.05 bps, the LSTM+MV performs better, with 1-percent CVaR of 0.0472, 5-percent VaR of 0.0196 and 5-percent CVaR of 0.0293. After including transaction cost $0.1 \mathrm{bps}$, in terms of 1 -percent $\mathrm{VaR}, \mathrm{SVM}+1 / \mathrm{N}$ model has the lowest value. LSTM+1/N achieves the lowest 1-percent VaR, SVM+1/N performs best for 5-percent VaR. return metrics on an annualised basis. It is clear that the LSTM+MV achieves the highest annualised returns of 0.1367 without transaction costs, followed by the SVM+MV (0.1022). SVM+MV and $\mathrm{SVM}+1 / \mathrm{N}$ perform best in terms of annualised mean returns with transaction cost 0.05 bps and 0.1 bps. The Sharpe ratio measures excess return using standard deviation and can be explained as the return per unit of risk. We find that the LSTM+MV achieves the highest level of 0.5845 , with the $\mathrm{SVM}+\mathrm{MV}$ coming in second with 0.4569 . After transaction cost $0.1 \mathrm{bps}$ and $0.05 \mathrm{bps}, \mathrm{SVM}+1 / \mathrm{N}$ gets the highest Sharpe ratio at 0.3294 and 0.3300 respectively. In addition, SVM+1/N achieves the first place in terms of standard deviation and Sortino ratio, followed by LSTM+MV (0.05 bps) and LSTM+1/N (0.1 bps) respectively. 

metrics. In order to compare these models further, we are thus able to choose these four more competitive strategies to visualize performance over time, i.e., from March 1994 to March 2019.

\subsubsection{Visualization on financial performance}

perform better in the previous section to display their performance for further comparisons. Besides,

614 of using machine learning for asset pre-selection and further verify whether our proposed method is effective comparing with other portfolio data sets. Fig. 6 presents the cumulative return for each model without transaction cost. The LSTM+MV model has an obviously higher result and achieves

617 cumulative return of 15.9 approximately. The profitability of the LSTM+1/N model follows, with 618 5.7, and then the SVM+MV, with 5.5. And the Random+MV and the SVM+1/N keep similar at about 3.3, the Random $+1 / \mathrm{N}$ is the lowest, with 2.5 . Furthermore, we should also figure out how the

LSTM+MV and other models behave at different levels of transaction costs.

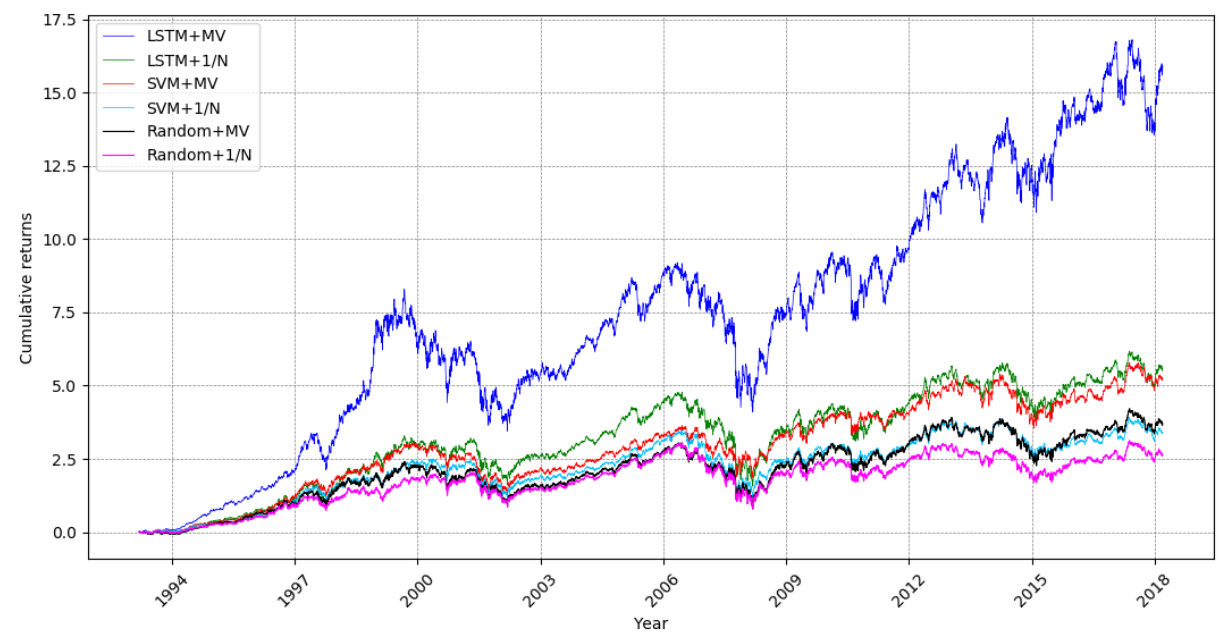

Fig. 6. cumulative return without transaction cost 

general, the LSTM+MV model still maintains a better accumulated return. The cumulative return with a transaction cost of $0.05 \mathrm{bps}$ is about 4.6 , while for a transaction cost of $0.10 \mathrm{bps}$ it is 4.5 .

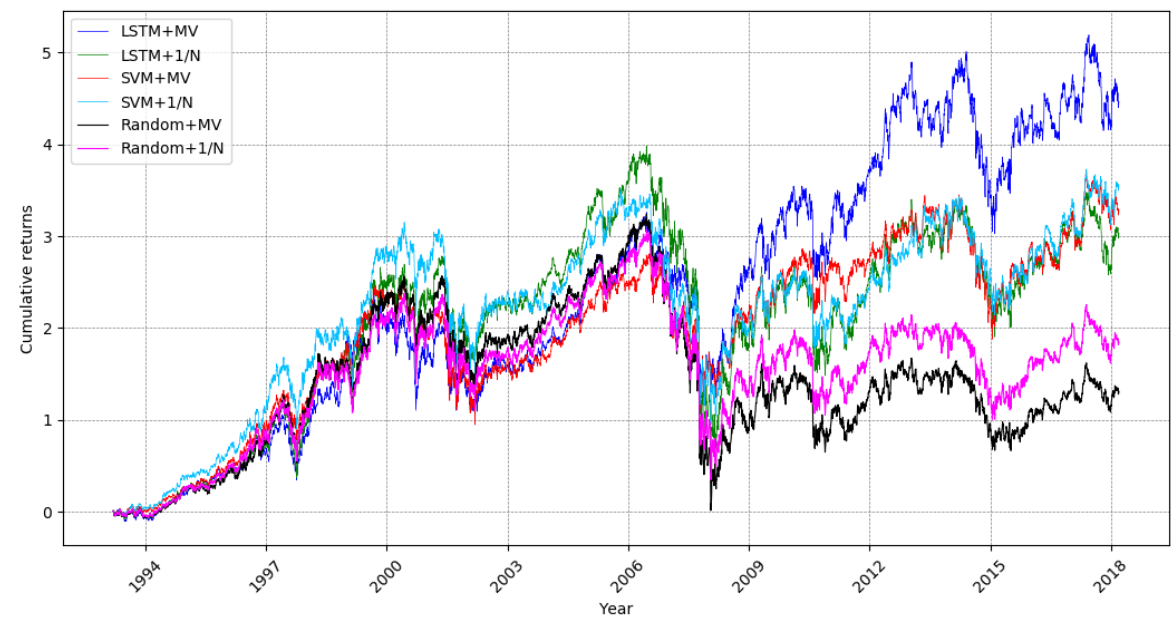

Fig. 7. cumulative return including transaction cost $(0.05 \mathrm{bps})$

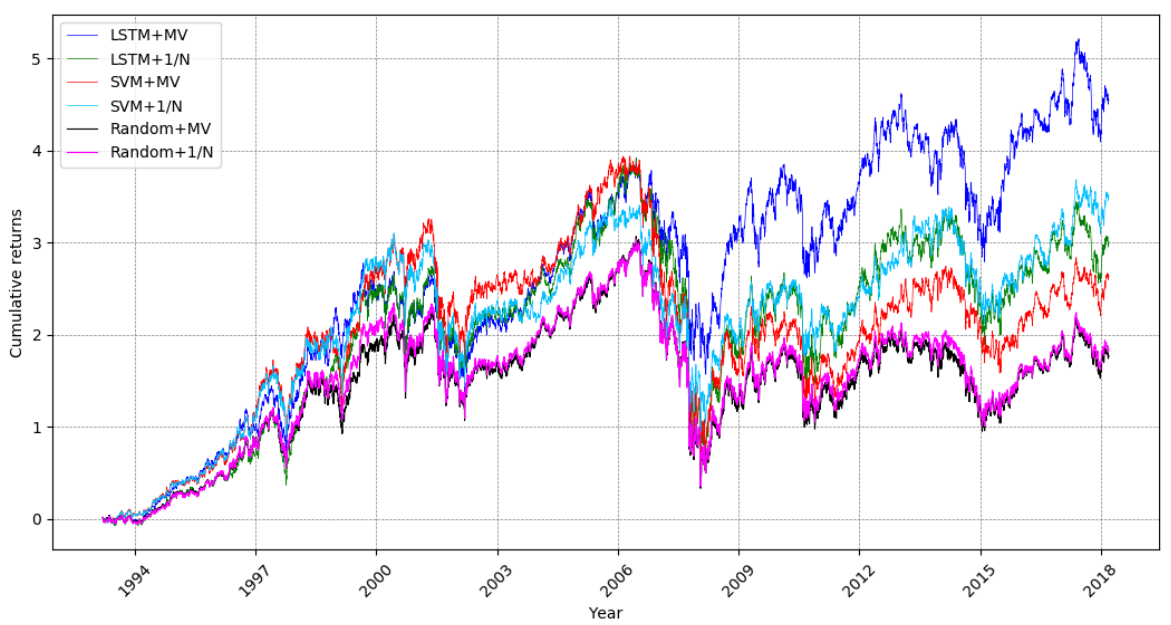

Fig. 8. cumulative return including transaction cost $(0.1 \mathrm{bps})$

From the comparison of the cumulative returns between the LSTM+MV model and the other baseline strategies, we can discover that LSTM+MV performs much better than other baselines in terms of return metrics. Another idea which is inspired from this is that we would like to see the results when integrating risks and whether the good performance only occurs during a certain period of time. As shown in Fig. 9, we use the Sharpe ratio performance of each model every three 
637 years. We can observe that, of the eight surveyed triennia, six of them show that the Sharpe ratio of the LSTM+MV model has a better result than other models during the corresponding periods.

639 Figures 10 and 11 present the Sharpe ratio per triennium with transaction costs. The LSTM+MV

640 model, with transaction costs of $0.05 \mathrm{bps}$, behaves better. Specifically, among the eight surveyed

641 triennia, five of them have a higher Sharpe ratio in LSTM+MV model than other models. After including transaction cost $0.1 \mathrm{bps}$, only half of the surveyed period shows a greater result of the

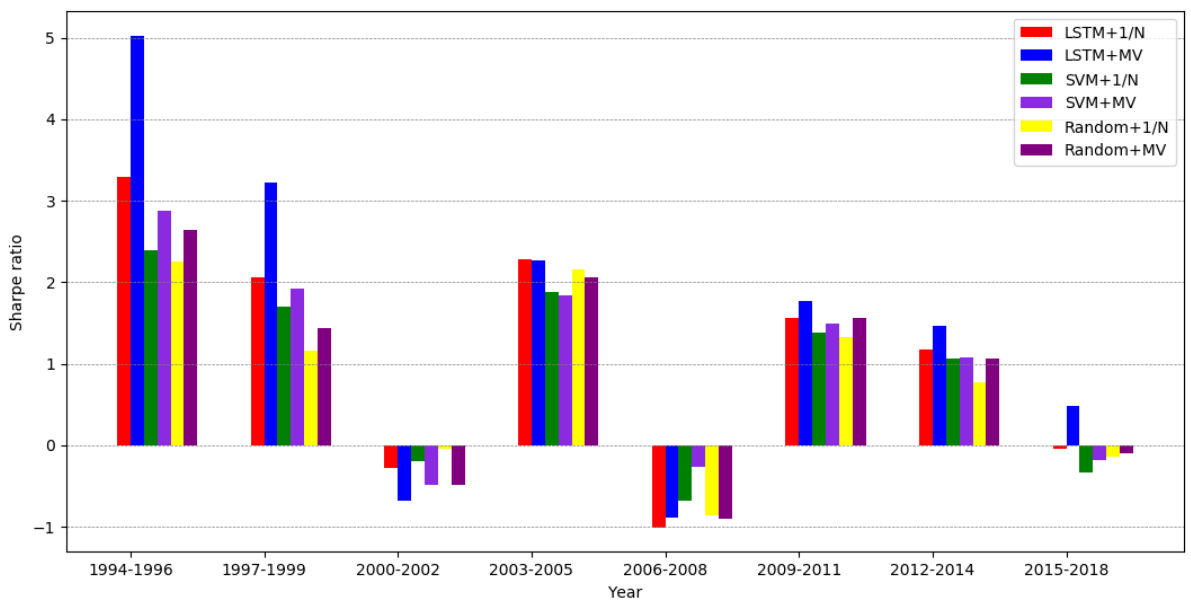

Fig. 9. Sharpe ratio of each triennium without transaction costs

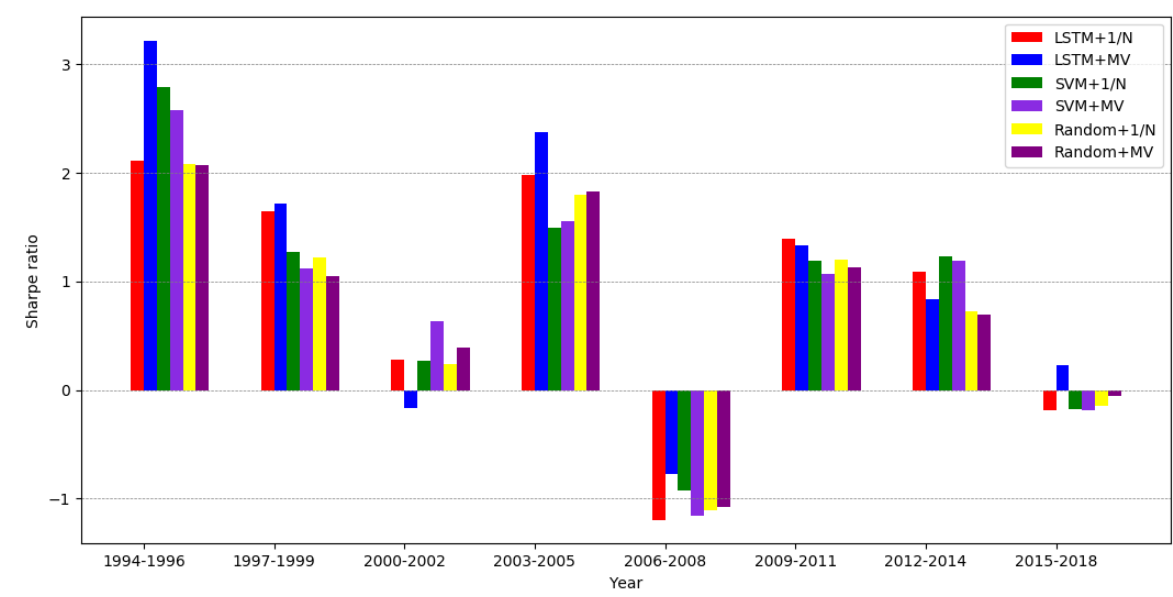

Fig. 10. Sharpe ratio of each triennium including transaction costs ( $0.05 \mathrm{bps})$ 


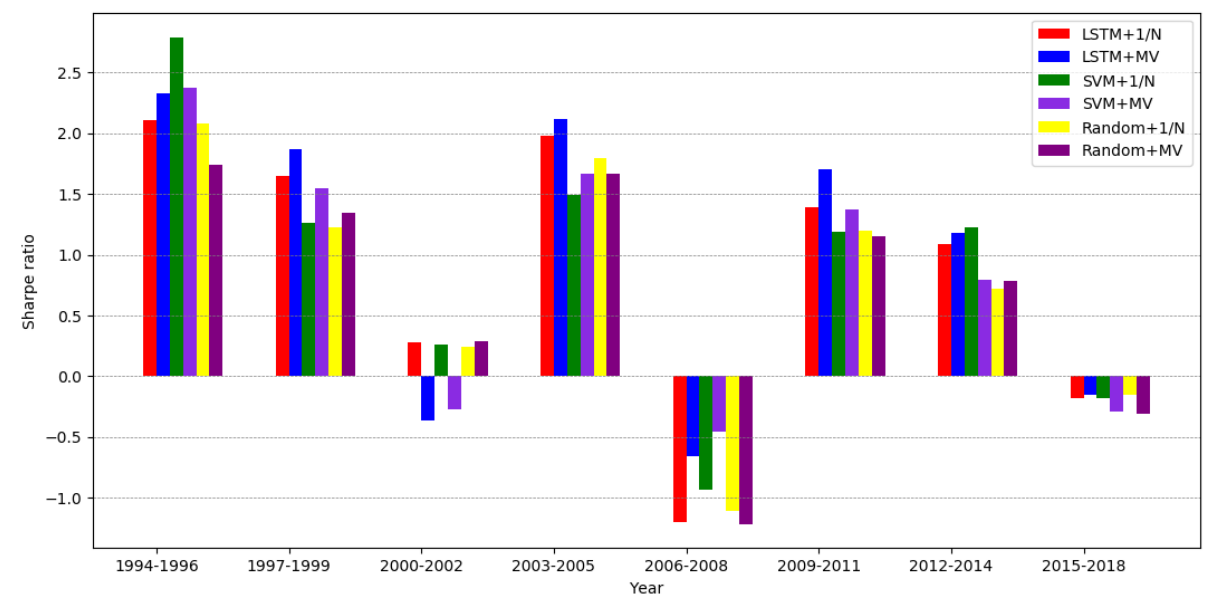

Fig. 11. Sharpe ratio of each triennium including transaction costs $(0.1 \mathrm{bps})$

Fig. 12 depicts the result of average return to the risk per month of each triennium per model without transaction costs. Apparently, the LSTM+MV model obtains a remarkable performance for the return-risk ratio during most study period. We also discover the average results as followings: 0.2670 for the LSTM+MV model, 0.1966 for the LSTM+1/N model, 0.1808 for the SVM+MV, 0.1581 for the $\mathrm{SVM}+1 / \mathrm{N}$ model, 0.1593 for the Random+MV, and 0.1458 for the Random $+1 / \mathrm{N}$ model. The LSTM+MV model stops having the highest value during period 2006-2008, and this result coincides with the financial crisis and troubled political.

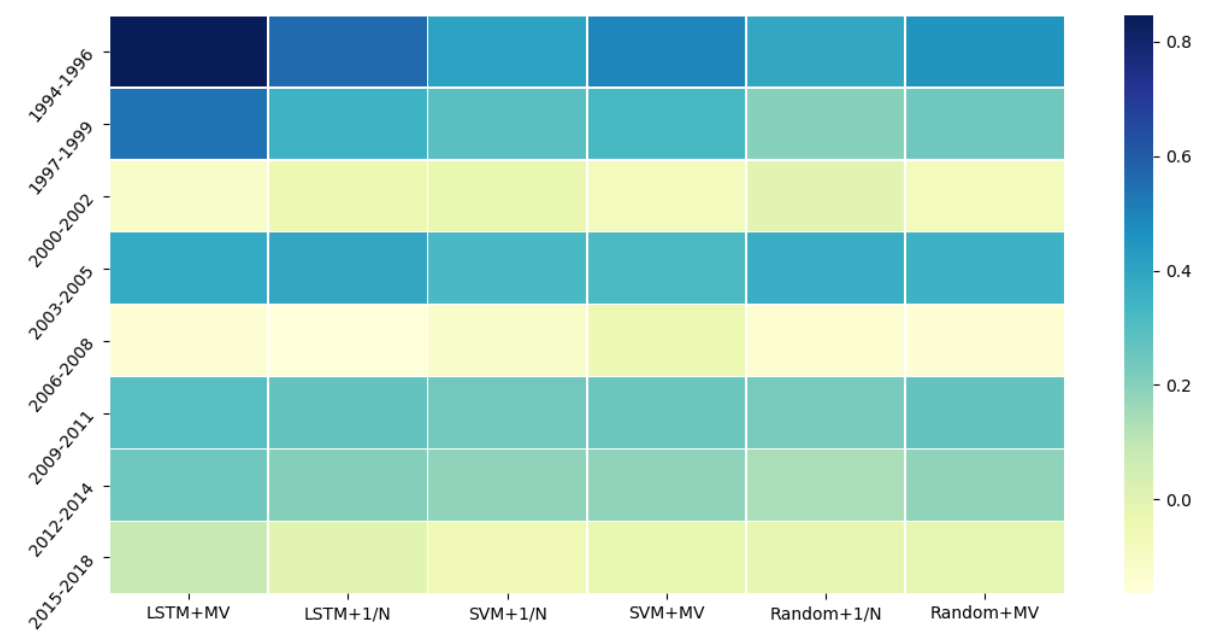

Fig. 12. Average return to the risk per month of each triennium without transaction costs

\section{Discussion and Conclusions}

\subsection{Discussion for key findings}

This paper puts forward an investment decision model entitled LSTM+MV. Based on the 
663 LSTM method, predict and select assets with a higher daily return of gain, then integrate this 664 prediction with the MV diversification method to compose the optimal portfolio. Our study results 665 in several important findings.

666 First of all, LSTM networks are applied to achieve the financial time-series prediction 667 empirical application on big data volume. Specifically, we create an appropriate prediction task, 668 divide whole sample data set into 22 overlapping training-testing sets, normalize the input features 669 in order to facilitate model training, find an appropriate LSTM architecture for forecasting. After 670 comparing the outcomes of the LSTM against SVM, RAF, DNN as well as ARIMA, we discover 671 the LSTM networks are appropriate for financial time-series forecasting, to beat the other early machine learning models and the statistics model by a very clear margin.

674 returns with the same level of risk. In this case, the LSTM+MV, SVM+MV, LSTM+1/N and $675 \mathrm{SVM}+1 / \mathrm{N}$ outperform the Random+MV and Random $+1 / \mathrm{N}$ in terms of the return, risk or risk-return metrics. Among these results, we further display their performance in accordance cumulative return 677 per year, Sharpe ratio per triennium as well as average return to the risk per month of each triennium. Finally, for cumulative return performance without transaction costs, the LSTM+MV model 679 is significantly better than the other baseline models. A three-year Sharpe ratio experiment also 680 confirms the better performance of the LSTM+MV model. After including transaction costs, the 681 LSTM+MV model still outperforms the other models with a better outcome. In that case, the applicability of the model's implementation may depend on the amount of money invested by investors. 


\subsection{Theoretical implications}

This research enriches the theoretical literature on the stock return prediction and portfolio management. First of all, the portfolio formation method proposed in this paper is able to capture the long-term dependences of financial time-series data fluctuation, which fills the gap in corresponding portfolio optimisation researches paying insufficient attention to the continuity and memory characteristics of financial time-series data. To be specific, this paper compares the forecasting outcomes of the LSTM with SVM, RAF, DNN as well as ARIMA to demonstrate the accuracy and feasibility of LSTM networks in predicting financial time-series more convincingly. Second, the preselection process of assets is incorporated into the optimal portfolio formation. Instead changing and improving the Markowitz' MV model, this paper puts effort into the preliminary phase of portfolio construction to ensure that the portfolio is composed of assets with high-return in the beginning. Specifically, our study demonstrates that the proposed model LSTM+MV is able to help individual investors obtain remarkable outcomes for the cumulative returns as well as risk-adjusted return for majority of periods. The merger of the return forecasting and portfolio optimisation processes may provide a new perspective for research in fintech area.

\subsection{Practical implications}

The study also provides several practical implications. For portfolio managers, this paper puts forwards a practical method for optimal portfolio selection that can help improve day investments. Following this model, managers can pick assets with higher return based on the predicting results in real market, and then apply MV model to reduce risk level so that keep investments safe and beneficial. For individual investors, this method is able to systematically help them to make decisions for investing. In another words, tell them which assets they should hold and how much to invest in each asset to achieve the goal of maximal potential return with minimal risk. 


\subsection{Limitations and future work}

Although this research provides useful insights, there are some limitations in this study, which provide opportunities for further research. First, five technical indicators and fifteen lagged variables are used as input features to predict the return in the future, however, there are some other external environment factors, such as government policies, interest rates, public events and so forth that have an impact on financial market can also be considered as the input indicators to the models

713 (Christou et al., 2017). In addition, the study uses the asset data in only one country of UK. Due to the different political environment and economic backgrounds, we cannot ensure whether the proposed method is suitable for the stock markets from other countries. Thus, in future research, asset data from more countries should be used for experiments and comparisons to further testify

717 the applicability and establish the boundaries of the proposed model.

\section{Acknowledgements}

Wuyu Wang, Weizi Li and Kecheng Liu conceptualised the idea; Wuyu Wang and Weizi Li completed the experiments; Wuyu Wang analysed the results and wrote the paper, Kecheng Liu,

Ning Zhang and Weizi Li provided language help and proof reading the paper.

\section{References}

Aggarwal, S., \& Aggarwal, S. (2017). Deep Investment in Financial Markets using Deep

Alexander, D. L. J., Tropsha, A., \& Winkler, D. A. (2015). Beware of $R^{2}$ : Simple, Unambiguous Assessment of the Prediction Accuracy of QSAR and QSPR Models. Journal of Chemical Information and Modeling, 55, 1316-1322.

Almahdi, S., \& Yang, S. Y. (2017). An adaptive portfolio trading system: A risk-return portfolio 
Arévalo, A., Niño, J., Hernández, G., \& Sandoval, J. (2016). High-frequency trading strategy based on deep neural networks. International conference on intelligent computing, 424436.

Baek, Y., \& Kim, H. Y. (2018). ModAugNet: A new forecasting framework for stock market index value with an overfitting prevention LSTM module and a prediction LSTM module. Expert Systems With Applications, 113, 457- 480.

Bao, W., Yue, J., \& Rao, Y. (2017). A deep learning framework for financial time series using stacked autoencoders and long-short term memory. PLoS ONE, 12(7), 1-24.

Bergstra, J., \& Bengio, Y. (2012). Random Search for Hyper-Parameter Optimization. Journal of Machine Learning Research, (3), 281-305.

Bodnar, T., Mazur, S., \& Okhrin, Y. (2017). Bayesian estimation of the global minimum variance portfolio. European Journal of Operational Research, 256, 292-307.

Bodnar, T., Parolya, T., \& Schmid, W. (2018). Estimation of the global minimum variance portfolio in high dimensions. European Journal of Operational Research, 266, 371-390.

Brooks, C., Rew, A. G., Ritson, S. (2001). A trading strategy based on the lead-lag relationship between the spot index and futures contract for the FTSE 100. International Journal of Forecasting, 17, 31-44.

Brown. D. B., \& Smith, J. E. (2011). Dynamic Portfolio Optimisation with Transaction Costs: Heuristics and Dual Bounds. Management Science, 57(10), 1752-1770.

Chen, Y. J., \& Hao, Y. J. (2018). Integrating principle component analysis and weighted support vector machine for stock trading signals prediction. Neurocomputing, (321), pp. 381- 402. 
752

753

754

755

756

757

758

759

760

761

762

763

764

765

766

767

768

769

770

771

772

773

Chong, E., Han, C., \& Park, F. C. (2017). Deep learning networks for stock market analysis and prediction: Methodology, data representations, and case studies. Expert Systems With Applications, (83), 187-205.

Chourmouziadis, K., \& Chatzoglou, P. D. (2016). An intelligent short term stock trading fuzzy system for assisting investors in portfolio management. Expert Systems with Applications, (43), 298-311.

Christou, C., Cunado, J., Gupta, R., \& Hassapis, C. (2017). Economic policy uncertainty and stock market returns in PacificRim countries: Evidence based on a Bayesian panel VAR model. Journal of Multinational Financial Management, (40), 92-102.

Chuluun, T. (2017). Global portfolio investment network and stock market comovement. Global Finance Journal, (33), 51- 68.

Deng, S. J., \& Min, X. (2013). Applied Optimization in Global Efficient Portfolio Construction Using Earning Forecasts, Journal of Investing, 22(4), 104-114.

Ding, X., Zhang, Y., Liu. T., \& Duan. J. (2015). Deep learning for event-driven stock prediction. Proceedings of the Twenty-Fourth International Joint Conference on Artificial Intelligence.

Drucker, H., Burges, C. J., Kaufman, L., Smola, A., \& Vapnik, V. (1997). Support Vector Regression Machines. Advances in Neural Information Processing Systems, 9, 155-161.

Fischer, T., \& Krauss, C. (2018). Deep learning with long short-term memory networks for financial market predictions. European Journal of Operational Research, 270, 654-669.

Gatev, E., Goetzmann, W. N., \& Rouwenhorst, K. G. (2006). Pairs trading: Performance of a relative-value arbitrage rule. Review of Financial Studies, 19(3), 797-827. 
774 Gandhmal, D. P., \& Kumar, K. (2019). Systematic analysis and review of stock market $775 \quad$ prediction techniques. Computer Science Review, 34, 100190

776 Grauer, R. R., \& Hakansson, N. H. (1993). On the Use of Mean-Variance and Quadratic Approximations in Implementing Dynamic Investment Strategies: A Comparison of Returns and Investment Policies. Management Science, 39(7), 856-871.

779

Graves, A., \& Schmidhuber, J. (2005). Framewise phoneme classification with bidirectional LSTM and other neural network architectures. Neural Networks, 18(5), 602-610.

Greff, K., Srivastava, R. K., Koutník, J., Steunebrink, B. R., \& Schmidhuber, J. (2017). LSTM: A Search Space Odyssey, IEEE Transactions on Neural Networks and Learning Systems, 28(10), 2222-22232.

Guerard Jr, J., B. Markowitz, H., \& Xu, G. L. (2015). Earnings forecasting in a global stock 785 selection model and efficient portfolio construction and management. International Journal of Forecasting, 31, 550-560.

Hinton, G. (2009). Deep belief networks. Scholarpedia, 4(5), 5947.

Hinton, G.E., \& Salakhutdinov, R. R. (2006). Reducing the Dimensionality of Data with Neural Networks. Science, 313(5786), 504-507.

Hochreiter, S., \& Schmidhuber, J. (1997). Long short-term memory. Neural computation, 9(8), 1735-1780.

792

Huang, C. F. (2012). A hybrid stock selection model using genetic algorithms and support 793 vector regression. Applied Soft Computing, 12, 807-818.

Jozefowicz, R., Zaremba, W., Sutskever, I. (2015). An empirical exploration of recurrent network architectures. In Proceedings of the 32nd International Conference on Machine 
Lachiheba, O., \& Gouiderb, M. S. (2018). A hierarchical Deep neural network design for stock returns prediction. Procedia Computer Science, (126), 264-272.

Längkvist, M., Karlsson, L. \& Loutfi, A. (2014). A Review of Unsupervised Feature Learning 800 and Deep Learning for Time - series Modeling. Pattern Recognition Letters, (42), 11-24.

LeCun, Y., Bengio, Y., \& Hinton, G. 2015. Deep Learning, Nature, 521(7553), 436- 444.

Li, J., \& Xu, J. (2013). Multi-objective portfolio selection model with fuzzy random returns and a compromise approach-based genetic algorithm. Information Sciences, 220, 507- 521.

Li, T., Zhang, W., \& Xu, W. (2013). Fuzzy possibilistic portfolio selection model with VaR 805 constraint and risk-free investment. Economic Modelling, 31, 12-17.

Li, T., Zhang, W., \& Xu, W. (2015). A fuzzy portfolio selection model with background risk. Applied Mathematics and Computation, 256, 505-513.

Li, Y., Yuan, Y. (2017). Convergence Analysis of Two-layer Neural Networks with ReLU Activation. Neural Information Processing Systems Conference.

Liu, H., \& Loewenstein, M. (2002). Optimal Portfolio Selection with Transaction Costs and Finite Horizons. The Review of Financial Studies Summer, 15(3), 805-835. movement prediction. Knowledge-Based Systems, (164), 163-173.

814 Loureiro, A.L.D., Miguéis, V.L., \& Silva, L. F. M. (2018). Exploring the use of deep neural networks for sales forecasting in fashion retail. Decision Support Systems, 114, 81-93.

Kara, Y., Boyacioglu, M. A., \& Baykan, O. K. (2011). Predicting direction of stock price index movement using artificial neural networks and support vector machines: The sample of 
819 Kao, L. J., Chiu, C. C., Lu, C. J., \& Yang, J. L. (2013). Integration of nonlinear independent component analysis and support vector regression for stock price forecasting. Neurocomputing, 99, 534- 542.

Kingma, D. P., \& Ba, J. (2014). Adam: A Method for Stochastic Optimization. CoRR, arXiv preprint arXiv:1412.6980.

Kocuk, B., \& Cornuéjols, G. (2018). Incorporating Black-Litterman views in portfolio construction when stock returns are a mixture of normals, Omega, In press.

Kolm, P. N., Tütüncü, R., \& Fabozzi, F. J. (2014). 60 Years of portfolio optimisation: Practical challenges and current trends. European Journal of Operational Research, (234), 356371.

Kraus, M., \& Feuerriegel, S. (2017). Decision support from financial disclosures with deep neural networks and transfer learning. Decision Support Systems, (104), 38-48.

Malkiel, B. G. (2007). A random walk down Wall Street: The time-tested strategy for successful investing. WW Norton \& Company.

Markowitz, H. (1952). Portfolio selection. Journal of Finance, 7(1), 77- 91.

Merton, R. C. (1969). Lifetime Portfolio Selection under Uncertainty: The Continuous-Time Case. The review of Economics and Statistics, 51(3), 247-257.

Paiva, F. D. A, Cardoso, R. T. N., Hanaoka, G. P., \& Duarte, W. M. (2019). Decision-making for financial trading: A fusion approach of machine learning and portfolio selection. Expert Systems with Applications, (115), 635-655. 

Expert Systems with Applications, (42), 259-268.

842 Pektas, A. O., \& Cigizoglu, H. K. (2013). ANN hybrid model versus ARIMA and ARIMAX models of runoff coefficient. Journal of Hydrology, 500, 21-36.

844 Rather, A. M. Agarwal, A., \& Sastry, V. N. (2015). Recurrent neural network and a hybrid model for prediction of stock returns. Expert Systems with Applications, 42, 3234-3241.

Ranguelova, E. (2001). Disposition Effect and Firm Size: New Evidence on Individual Investor Trading Activity. SSRN eLibrary.

Reimers, N., \& Gurevych, I. (2017). Optimal Hyperparameters for Deep LSTM-Networks for Sequence Labeling Tasks. CoRR, arXiv preprint arXiv:1707.06799.

Sadaei, H. J., Enayatifar, R., Lee, M. H., \& Mahmud, M. (2016). A hybrid model based on differential fuzzy logic relationships and imperialist competitive algorithm for stock

Santos, A. A. P., \& Tessari, C. (2012). Quantitative portfolio optimisation techniques applied

Sharpe, W. F. (1963). A Simplified Model for Portfolio Analysis. Management Science, 9(2), 277-293.

Shmueli, G. (2010). To Explain or to Predict? Statistical Science, 25(3), 289-310.

Srivastava, N., Hinton, G., Krizhevsky, A., Sutskever, I., \& Salakhutdinov, R. (2014). Dropout: Learning Research, 15, 1929-1958.

861 Tanaka, H., Guoa, P., Turksen, B. (2000). Portfolio selection based on fuzzy probabilitiesand 
863 Ticknor, J. L. (2013). A Bayesian regularized artificial neural network for stock market 864 forecasting. Expert Systems with Applications, 40 (14), 5501-5506.

865 Tobin, J. (1958). Liquidity Preference as Behavior Towards Risk. The Review of Economic $866 \quad$ Studies, 25(2), 65-86.

867 Tu, J., Zhou, G. (2010). Incorporating Economic Objectives into Bayesian Priors: Portfolio 868 Choice under Parameter Uncertain. Journal of Financial and Quantitative Analysis, 45(4), 869 959-986.

870 Weng, B., Lu, L., Wang, X., Megahed, F. M., \& Martinez, W. (2018). Predicting short-term stock prices using ensemble methods and online data sources. Expert Systems With

Yunusoglu, M. G., \& Selim, H. (2013). A fuzzy rule based expert system for stock evaluation and portfolio construction: An application to Istanbul Stock Exchange. Expert Systems with Applications, (40), 908-920.

Zbikowski, K. (2015). Using Volume Weighted Support Vector Machines with walk forward testing and feature selection for the purpose of creating stock trading strategy. Expert Systems with Applications, 42, 1797-1805.

Zhang, Y., Li, X., \& Guo, S. (2018). Portfolio selection problems with Markowitz's meanvariance framework: a review of literature. Fuzzy Optimal Decision Making, 17, 125-158. 